\title{
Maximal width of the separatrix chaotic layer
}

\author{
S. M. Soskin ${ }^{1,2,3}$ and R. Mannella ${ }^{4}$ \\ ${ }^{1}$ Institute of Semiconductor Physics, National Academy of Sciences of Ukraine, 03028 Kiev, Ukraine \\ ${ }^{2}$ Abdus Salam ICTP, 34100 Trieste, Italy \\ ${ }^{3}$ Physics Department, Lancaster University, Lancaster LA1 4YB, United Kingdom \\ ${ }^{4}$ Dipartimento di Fisica, Università di Pisa, 56127 Pisa, Italy
}

(Received 1 July 2008; revised manuscript received 9 November 2009; published 29 December 2009)

\begin{abstract}
The main goal of the paper is to find the absolute maximum of the width of the separatrix chaotic layer as function of the frequency of the time-periodic perturbation of a one-dimensional Hamiltonian system possessing a separatrix, which is one of the major unsolved problems in the theory of separatrix chaos. For a given small amplitude of the perturbation, the width is shown to possess sharp peaks in the range from logarithmically small to moderate frequencies. These peaks are universal, being the consequence of the involvement of the nonlinear resonance dynamics into the separatrix chaotic motion. Developing further the approach introduced in the recent paper by Soskin et al. [Phys. Rev. E 77, 036221 (2008)], we derive leading-order asymptotic expressions for the shape of the low-frequency peaks. The maxima of the peaks, including in particular the absolute maximum of the width, are proportional to the perturbation amplitude times either a logarithmically large factor or a numerical, still typically large, factor, depending on the type of system. Thus, our theory predicts that the maximal width of the chaotic layer may be much larger than that predicted by former theories. The theory is verified in simulations. An application to the facilitation of global chaos onset is discussed.
\end{abstract}

DOI: 10.1103/PhysRevE.80.066212

PACS number(s): 05.45.Ac, 05.40.- a, 05.45.Pq

\section{INTRODUCTION}

Separatrix chaotic layers (SCLs) play a fundamental role for Hamiltonian chaos and may be important in a broad variety of subjects in physics and astronomy [1-11]. One of the most important characteristics of the layer is its width in energy or in related quantities. It can be easily found numerically by means of integration of the Hamiltonian equations with a set of initial conditions in the vicinity of the separatrix. But it is important also to be able to predict it theoretically. There is a long and rich history of the corresponding studies. The results may be classified as follows.

\section{A. Heuristic analytic results}

Consider a one-dimensional (1D) Hamiltonian system perturbed by a weak time-periodic perturbation

$$
\begin{gathered}
H=H_{0}(p, q)+h V(p, q, t), \\
V\left(p, q, t+2 \pi / \omega_{f}\right)=V(p, q, t), \quad h \ll 1,
\end{gathered}
$$

where $H_{0}(p, q)$ possesses a separatrix and, for the sake of notation compactness, all relevant parameters of $H_{0}$ and $V$, except possibly $\omega_{f}$, are assumed to be $\sim 1$.

There were a few heuristic criteria set by physicists (see, e.g., [1-6]) which gave qualitatively similar results for the SCL width $\Delta E$ in terms of energy $E \equiv H_{0}(p, q)$,

$$
\begin{gathered}
\Delta E \equiv \Delta E\left(\omega_{f}\right) \sim \omega_{f} \delta, \\
\delta \equiv h|\epsilon|, \\
|\epsilon| \lesssim 1 \quad \text { for } \quad \omega_{f} \lesssim 1, \\
|\epsilon| \propto \exp \left(-a \omega_{f}\right) \ll 1 \quad(a \sim 1) \quad \text { for } \quad \omega_{f} \gg 1 .
\end{gathered}
$$

The quantity $\delta \equiv h|\epsilon|$ is called the separatrix split [5] [see also Eq. (4) below]: it determines the maximum distance between the perturbed incoming and outgoing separatrices [1-9]. It follows from Eq. (2) that the maximum of $\Delta E$ lies in the frequency range $\omega_{f} \sim 1$ while the maximum itself is $\sim h$,

$$
\Delta E_{\max } \equiv \max _{\omega_{f}}\left\{\Delta E\left(\omega_{f}\right)\right\} \sim h, \quad \omega_{f}^{(\max )} \sim 1 .
$$

\section{B. Mathematical and accurate physical results}

Many papers studied the SCL by mathematical and accurate physical methods. For the range $\omega_{f} \gg 1$, there are many works studying the separatrix splitting (see the review [8] and references therein) and the SCL width in terms of the normal coordinates (see the review [9] and references therein). Though quantities studied in these works typically differ from those studied by physicists [1-6], they implicitly confirm the main qualitative conclusion from the heuristic formula (2) in the high-frequency range: if $\omega_{f} \gg 1$, the SCL width is exponentially small.

There are also several works studying the SCL in the opposite (i.e., adiabatic) limit $\omega_{f} \rightarrow 0$ (see, e.g., [12-16] and references therein). In the context of the SCL width, it is most important that $\Delta E\left(\omega_{f} \rightarrow 0\right) \sim h$ for most of the systems [12-14]. For a particular class of systems, namely, for acdriven spatially periodic systems (e.g., the ac-driven pendulum), the width of the SCL part above the separatrix diverges in the adiabatic limit $[15,16]$ : the divergence develops for $\omega_{f} \ll 1 / \ln (1 / h)$.

Finally, there is a qualitative estimation of the SCL width for the range $\omega_{f} \sim 1$ within the Kolmogorov-Arnold-Moser (KAM) theory [9] while the quantitative estimation within the KAM theory appears to be very difficult for this fre- 
quency range [17]. It follows from the results in [9] that the width in this range is of the order of the separatrix split while the latter is of the order of $h$.

Thus, from the above results, it appears to follow that, for all systems except the ac-driven spatially periodic systems, the maximum of the SCL width is $\sim h$ and occurs in the range $\omega_{f} \sim 1$, quite in agreement with the heuristic result (3). Even for the ac-driven spatially periodic systems, this conclusion appears to apply to the width of the SCL part below the separatrix, for the whole frequency range, and to the width of the SCL part above the separatrix, for $\omega_{f}$ $\gtrsim 1 / \ln (1 / h)$.

\section{Numerical evidences of high peaks in $\Delta E\left(\omega_{f}\right)$ and their rough analytic estimates}

The above conclusion does not agree with several numerical studies carried out during the last decade (see, e.g., $[15,16,18-23])$ which have revealed the existence of sharp peaks in $\Delta E\left(\omega_{f}\right)$ in the frequency range $1 / \ln (1 / h) \lesssim \omega_{f} \lesssim$ $\sim 1$, the heights of which greatly exceed $h$ (see also Figs. 2, 3,5 , and 6). Thus, the peaks represent the general dominant feature of the function $\Delta E\left(\omega_{f}\right)$. The peaks were related by the authors of [18-23] to the absorption of nonlinear resonances by the SCL.

The first theory related to these peaks was developed in [18]: for a partial model, it gave heuristic analytic estimates for the positions of the maxima of the peaks (see also [23], where the positions of the maxima were estimated for the same model using a different method) and for their heights. While the positions predicted by the theories [18,23] are in a reasonable agreement with computer simulations in a wide range of parameters, the estimate for the heights [18] is inaccurate in many ranges of parameters, in particular in the asymptotic limit $h \rightarrow 0$ (for a more detailed discussion, see Sec. V B below). The mechanism based on the involvement of nonlinear resonances into the separatrix chaos is introduced in [18] on a solely heuristic base while, in the asymptotic limit $h \rightarrow 0$, the mechanism is more complicated [24,25].

\section{Approach for the accurate description of the peaks}

It is explicitly stated in the review [21] that the search for the mechanism of the involvement of resonances into the separatrix chaos and the accurate analytic description of the peaks are among the most important and challenging tasks in the study of separatrix chaos. The first step toward this accomplishment was done in the recent papers [24,25], where a new approach to the theoretical treatment of the separatrix chaos for the relevant frequency range was developed and applied to the problem of the onset of global chaos between two close separatrices. An application of the approach to the, more common, single-separatrix cases was only briefly discussed in [24,25].

The present paper formulates the basic ideas of the approach in terms more general than $[24,25]$ and, on the basis of this approach, develops an accurate asymptotic description of the peaks, i.e., of the SCL width as a function of frequency in the range of the maximum of the width, which is the most important range from a physical point of view. In particular, we show that the maximal width of the separatrix chaotic layer may be much larger than it was assumed before. We classify all systems into two different types: for systems of type I, the ratio between the maximal width and the perturbation amplitude $h$ logarithmically diverges in the asymptotic limit $h \rightarrow 0$ while, for systems of type II, it asymptotically approaches a constant (still large, typically).

Though the form of our treatment differs from typical forms of mathematical theorems in this subject (cf. [8,9]), the results yield the exact leading-order term in the asymptotic expansion of the width in the parameter of smallness $\alpha \equiv 1 / \ln (1 / h)$. Our theory is in a good agreement with the results of numerical integration of the equations of motion.

Section II describes the basic ideas of the approach. Section III presents the classification into two types of systems using rough estimates. Section IV develops the leading-order asymptotic theory for an archetypal example of type I and compares it to the numerical integration of Hamiltonian equations of motion. Section V develops the leading-order asymptotic theory for two archetypal examples of type II and compares it to the numerical integration. Next-order corrections are estimated in Sec. VI. Discussion of a few other issues, including in particular an application to the global chaos onset, is presented in Sec. VII. Conclusions are drawn in Sec. VIII.

\section{BASIC IDEAS OF THE APPROACH}

The approach, which is developed in $[24,25]$ and here, may be briefly formulated as the matching between the discrete chaotic dynamics of the separatrix map in the immediate and moderate vicinity of the separatrix and the continuous regularlike dynamics of the resonance Hamiltonian in the moderate and far vicinity of the separatrix (the terms "immediate, moderate, and far vicinity" will be clear from the further consideration). The present section describes the general features of the approach in more details.

The motion near the separatrix may be approximated by the separatrix map (SM) [1-7,9,18,23-26]. It was introduced for the first time in [1] and its various modifications were used in many studies afterwards, sometimes being called as the whisker map. It was rederived in [26] rigorously, as the leading-order approximation of the motion near the separatrix in the asymptotic limit $h \rightarrow 0$, and an estimate of the errors was carried out in [26] too (see also [9] and references therein).

We remind the main ideas which allow one to introduce the SM [1-7,9,24-26]. For the sake of simplicity, let us consider a perturbation $V$ that does not depend on the momentum: $V \equiv V(q, t)$. A system with an energy close to the separatrix value spends most of the time in the vicinity of the saddle(s), where the velocity is exponentially small. Differentiating $E \equiv H_{0}(p, q)$ with respect to time and allowing for the equations of motion of the system (1), we can show that $\dot{E}=\dot{q} \partial V / \partial q \propto \dot{q}$. Thus, the perturbation can significantly change the energy only when the velocity is not small, i.e., during the relatively short intervals while the system is away 
from the saddle(s): these intervals correspond to pulses of velocity as a function of time. Consequently, it is possible to approximate the continuous Hamiltonian dynamics by a discrete dynamics which maps the energy $E$, the perturbation angle $\varphi \equiv \omega_{f} t$, and the velocity $\operatorname{sign} \sigma \equiv \operatorname{sgn}(\dot{q})$ from pulse to pulse.

The actual form of the SM may vary, depending on the system under study, but its features, relevant in the present context, are similar for all systems. For the sake of clarity, let us consider the more explicit case when the separatrix of $H_{0}(p, q)$ possesses a single saddle and two symmetric loops while $V=q \cos \left(\omega_{f} t\right)$. Then the SM reads [24]

$$
\begin{gathered}
E_{i+1}=E_{i}+\sigma_{i} h \epsilon \sin \left(\varphi_{i}\right), \\
\varphi_{i+1}=\varphi_{i}+\frac{\omega_{f} \pi\left[3-\operatorname{sgn}\left(E_{i+1}-E_{s}\right)\right]}{2 \omega\left(E_{i+1}\right)}, \\
\sigma_{i+1}=\sigma_{i} \operatorname{sgn}\left(E_{s}-E_{i+1}\right), \quad\left|\sigma_{i}\right|=1, \\
\epsilon \equiv\left(\omega_{f}\right)=\left.\operatorname{sgn}\left(\left.\frac{\partial H_{0}}{\partial p}\right|_{t \rightarrow-\infty}\right) \int_{-\infty}^{\infty} d t \frac{\partial H_{0}}{\partial p}\right|_{E_{s}} \sin \left(\omega_{f} t\right), \\
\left.E_{i} \equiv H_{0}(p, q)\right|_{t_{i}-\Delta}, \\
\varphi_{i} \equiv \omega_{f} t_{i}, \\
\sigma_{i} \equiv \operatorname{sgn}\left(\left.\frac{\partial H_{0}}{\partial p}\right|_{t_{i}}\right),
\end{gathered}
$$

where $E_{s}$ is the separatrix energy, $\omega(E)$ is the frequency of oscillation with energy $E$ in the unperturbed case (i.e., for $h=0), t_{i}$ is the instant corresponding to the $i$ th turning point in the trajectory $q(t)$, and $\Delta$ is an arbitrary value from the range of time intervals which greatly exceed the characteristic duration of the velocity pulse while being much smaller than the interval between the subsequent pulses $[1-7,9,26]$.

Consider the two most general ideas of our approach:

(1) If a trajectory of the SM includes a state with $E=E_{s}$ and an arbitrary $\varphi$ and $\sigma$, then this trajectory is chaotic. Indeed, the angle $\varphi$ of such a state is not correlated with the angle of the state at the previous step of the map due to the divergence of $\omega^{-1}\left(E \rightarrow E_{s}\right)$. Therefore, the angle at the previous step may deviate from a multiple of $2 \pi$ by an arbitrary value and, hence, the energy of the state at the previous step may deviate from $E_{s}$ by an arbitrary value within the interval $[-h|\epsilon|, h|\epsilon|]$. The velocity sign $\sigma$ is not correlated with that at the previous step either [27]. Given that a regular trajectory of the SM cannot include a step where all three variables of the SM change randomlike, we conclude that such a trajectory is chaotic.

Though the above arguments appear to be obvious, they may not be considered as a mathematically rigorous proof, so that the statement about the chaotic nature of the SM trajectory which includes any state with $E=E_{s}$ should be considered as a conjecture supported by the above arguments and by the results of the numerical iteration of the SM. Possibly, the mathematically rigorous proof should involve an analysis of the Lyapunov exponents for the SM (cf. [3]) but this appears to be a technically difficult problem. We emphasize however that the rigorous proof of the conjecture is not crucial for the validity of the main results of the present paper, namely, of the leading terms in the asymptotic expressions describing the low-frequency peaks of the SCL width as a function of the perturbation frequency.

(2) As well known [1-9,18,23-25], at the leading-order approximation, the frequency of eigenoscillation as function of the energy near the separatrix is proportional to the reciprocal of the logarithmic factor. For example, for $H_{0}$ relevant to Eq. (4),

$$
\begin{gathered}
\omega(E) \propto \frac{b}{\ln \left(\frac{1}{\left|E-E_{s}\right|}\right)}, \quad b=\frac{3-\operatorname{sgn}\left(E-E_{s}\right)}{2}, \\
\left|E-E_{s}\right| \ll 1 .
\end{gathered}
$$

Given that the argument of the logarithm is large in the relevant range of $E$, the function $\omega(E)$ is nearly constant for a substantial variation of the argument. Therefore, as the SM maps the state $\left(E_{0}=E_{s}, \varphi_{0}, \sigma_{0}\right)$ onto the state with $E=E_{1}$ $\equiv E_{s}+\sigma_{0} h \epsilon \sin \left(\varphi_{0}\right)$, the value of $\omega(E)$ for the given $\operatorname{sgn}\left[\sigma_{0} \epsilon \sin \left(\varphi_{0}\right)\right]$ is nearly the same for most of the angles $\varphi_{0}$ (except in the close vicinity of multiples of $\pi$ ), namely,

$$
\begin{gathered}
\omega(E) \approx \omega_{r}^{( \pm)}, \\
\omega_{r}^{( \pm)} \equiv \omega\left(E_{s} \pm h\right), \quad \operatorname{sgn}\left[\sigma_{0} \epsilon \sin \left(\varphi_{0}\right)\right]= \pm 1 .
\end{gathered}
$$

Moreover, if the deviation of the SM trajectory from the separatrix increases further, $\omega(E)$ remains close to $\omega_{r}^{( \pm)}$ provided the deviation is not too large, namely, if $\ln \left(\left|E-E_{s}\right| / h\right) \ll \ln (1 / h)$. If $\omega_{f} \lesssim \omega_{r}^{( \pm)}$, then the evolution of the SM may be regularlike for a long time until the energy returns to the close vicinity of the separatrix, where the trajectory is chaotized. Such a behavior is especially pronounced if the perturbation frequency is close to $\omega_{r}^{(+)}$or $\omega_{r}^{(-)}$ or to one of their multiples of relatively low order: the resonance between the perturbation and the eigenoscillation gives rise to an accumulation of energy changes for many steps of the SM, which results in a deviation of $E$ from $E_{s}$ that greatly exceeds the separatrix split $h|\epsilon|$. Consider a state at the boundary of the SCL. The deviation of energy of such a state from $E_{s}$ depends on its position at the boundary. In turn, the maximum deviation is a function of $\omega_{f}$. The latter function possesses the absolute maximum at $\omega_{f}$ close to $\omega_{r}^{(+)}$ or $\omega_{r}^{(-)}$typically [28], for the upper or lower boundary of the SCL, respectively. This corresponds to the involvement of the, respectively, upper and lower, first-order nonlinear resonance into the separatrix chaos.

The above intuitive idea has been explicitly confirmed in [24]. It has been shown in the Appendix of [24] that, in the relevant range of energies, the $\mathrm{SM}$ can be reduced to the system of two differential equations which are identical to the equations of motion of the auxiliary resonance Hamiltonian which describes the resonance dynamics in terms of the conventional canonically conjugate slow variables, action $I$, and slow angle $\widetilde{\psi} \equiv n \psi-\omega_{f} t$, where $\psi$ is the angle variable 
[2-7] (see Eq. (16) below) while $n$ is the relevant resonance number, i.e., the integer number closest to the ratio $\omega_{f} / \omega_{r}^{( \pm)}$.

Thus, the result of the matching between the discrete chaotic dynamics of the SM and the continuous regularlike dynamics of the resonance Hamiltonian is the following [24]. After the chaotic trajectory of the SM visits any state on the separatrix, the system transits in one step of the SM to a given upper or lower curve in the $I-\widetilde{\psi}$ plane which has been labeled [24], respectively, upper or lower generalized separatrix split (GSS) curve [29]

$$
E=E_{\mathrm{GSS}}^{( \pm)}(\widetilde{\psi}) \equiv E_{s} \pm \delta|\sin (\widetilde{\psi})|, \quad \delta \equiv h|\epsilon|,
$$

where $\delta$ is the conventional separatrix split [5] while $\epsilon$ is the amplitude of the Melnikov-like integral [1-9,18,22-25] [for the separatrix of $H_{0}$ relevant to Eq. (4), $\epsilon$ is defined in Eq. (4)] and the angle $\tilde{\psi}$ may take any value from one of the two ranges: either $[0, \pi]$ or $[\pi, 2 \pi][30]$.

After that, because of the closeness of $\omega_{f} \approx n \omega_{r}^{ \pm}$to the $n$th harmonic of $\omega(E)$ in the relevant range of $E$ [31], for a relatively long time the system follows the nonlinear resonance (NR) dynamics [see Eq. (16) below] during which the deviation of the energy from the separatrix value grows, greatly exceeding $\delta$ for most of the trajectory. As time goes on, $\tilde{\psi}$ is moving and, at some point, the deviation in energy from the separatrix value begins to decrease. This decrease lasts until the system hits the GSS curve, after which it returns to the separatrix just for one step of the SM. At the separatrix, the slow angle $\tilde{\psi}$ is chaotized, so that a new stage of evolution similar to the one just described occurs, i.e., the system transits to the GSS curve with a new (random) value of $\tilde{\psi}$ and then the NR dynamics starts.

Of course, the SM cannot describe the variation of the energy during the velocity pulses [more exactly, in between instants $t_{i}-\Delta$ and $t_{i+1}-\Delta$, relevant to energies $E_{i}$ and $E_{i+1}$, respectively: see Eq. (4) and the definitions of $t_{i}$ and $\Delta$ following Eq. (4)]: in some cases, this variation can be comparable to the change within the SM dynamics. This additional variation will be taken into account, where relevant (see Sec. $\mathrm{V}$ below).

One might argue that, even for the instants relevant to the SM, the SM describes the original Hamiltonian dynamics only approximately [26] and may therefore miss some fine details of the motion: for example, the above picture does not include small windows of stability on the very separatrix. However, these fine details are irrelevant in the present context, in particular the relative portion of the windows of stability on the separatrix apparently vanishes in the asymptotic limit $h \rightarrow 0$.

The boundary of the SM chaotic layer is formed by those parts of the SM chaotic trajectory which deviate from the separatrix more than others. As follows from the structure of the chaotic trajectory described above, the lower boundary of the SM chaotic layer is formed in one of the two following ways [24,25]: (1) if there exists a self-intersecting resonance trajectory (in other words, the resonance separatrix), the upper part of which (i.e., the part situated above the selfintersection) touches or intersects the lower GSS curve while
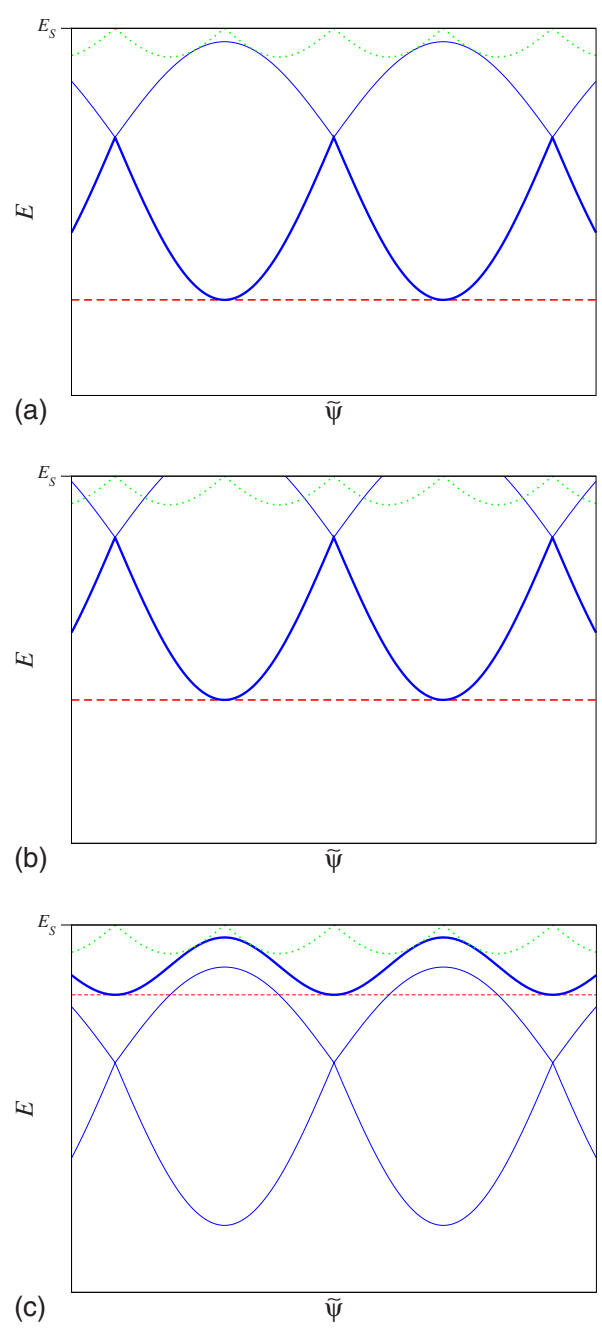

FIG. 1. (Color online) A schematic figure illustrating the formation of the peak of the function $\Delta E_{s m}^{(-)}\left(\omega_{f}\right)$ : (a) $\omega_{f}=\omega_{\max }$; (b) $\omega_{f}$ $<\omega_{\max }$; (c) $\omega_{f}>\omega_{\max }$. The relevant (lower) GSS curve is shown by the dotted line. The relevant trajectories of the resonance Hamiltonian are shown by solid lines. The lower boundary of the layer is marked by a thick solid line: in (a) and (b) the lower boundary is formed by the lower part of the resonance separatrix while, in (c) it is formed by the resonance trajectory tangent to the GSS curve. Dashed line marks, for a given $\omega_{f}$, the maximal deviation of the lower boundary from the separatrix energy $E_{s}$ i.e., $\Delta E_{s m}^{(-)}\left(\omega_{f}\right)$.

the lower part does not, then the lower boundary of the layer is formed by the lower part of this self-intersecting trajectory [Figs. 1(a) and 1(b)]; (2) otherwise the boundary is formed by the resonance trajectory tangent to the GSS curve [Fig. $1(\mathrm{c})]$. The upper boundary of the SM chaotic layer is formed analogously.

It is shown below that the variation of the energy along the resonance trajectory forming the boundary is larger than the separatrix split $\delta$ by a logarithmically large factor $\propto \ln (1 / h)$. Therefore, over the boundary of the SM chaotic layer, the largest deviation of the energy from the separatrix value, $\Delta E_{s m}^{( \pm)}$, may be taken, in the leading-order approximation, to be equal to the largest variation of the energy along the resonance trajectory forming the boundary, while the latter trajectory can be entirely described within the resonance Hamiltonian formalism. 
Finally, we mention in this section that, as obvious from the above description of the boundary, $\Delta E_{s m}^{( \pm)} \equiv \Delta E_{s m}^{( \pm)}\left(\omega_{f}\right)$ possesses a local maximum $\Delta E_{\max , s m}^{( \pm)}$at $\omega_{f}$ for which the resonance separatrix just touches the corresponding GSS curve [see Fig. 1(a)].

\section{ROUGH ESTIMATES: CLASSIFICATION OF SYSTEMS}

As obvious from Sec. II above, $\Delta E_{\max , s m}^{( \pm)}$is equal, in the leading order, to the width $\Delta E_{\mathrm{NR}}$ of the nonlinear resonance which touches the separatrix chaotic layer. Let us make a rough estimate of $\Delta E_{\mathrm{NR}}$ : it will allow us to classify all systems into two different types. With this aim, we expand the perturbation $V$ into a Fourier series in $t$ and a Fourier series in $\psi$,

$$
\begin{aligned}
V & \equiv \frac{1}{2} \sum_{l} V^{(l)}(E, \psi) \exp \left(-i l \omega_{f} t\right)+\text { c.c. }, \\
& \equiv \frac{1}{2} \sum_{l, k} V_{k}^{(l)}(E) \exp \left[i\left(k \psi-l \omega_{f} t\right)\right]+\text { c.c. }
\end{aligned}
$$

As in the standard theory of a nonlinear resonance [2-6], let us single out the relevant $V_{K}^{(L)}$ for a given peak (i.e., $\left.K \omega_{r}^{( \pm)} \approx L \omega_{f}\right)$ and denote its absolute value by $V_{0}$,

$$
V_{0}(E) \equiv\left|V_{K}^{(L)}(E)\right| .
$$

Let us now roughly estimate the width of the resonance using the pendulum approximation of the resonance dynamics [2-7]

$$
\Delta E_{\mathrm{NR}} \sim \sqrt{\frac{8 h V_{0} \omega_{f}}{|d \omega / d E|}} .
$$

Of course, this approximation assumes the approximate constancy of $V_{0}$ and $d \omega / d E$ in the resonance range of energies, while it is not so in our case, at least for $d \omega / d E$ : indeed, $\omega(E) \propto 1 / \ln \left(1 /\left|E-E_{s}\right|\right)$ in the vicinity of the separatrix $[1-7,9,18,22-25]$, so that the absolute value of the relevant derivative $|d \omega / d E| \sim\left(\omega_{r}^{( \pm)}\right)^{2} /\left|E-E_{s}\right|$ strongly varies within the resonance range. As a result, the shape of the nonlinear resonance separatrix is strongly asymmetric (cf. Fig. 4 below). However, for our rough estimate, we may use the maximal value of $\left|E-E_{s}\right|$, which is equal to $\Delta E_{\mathrm{NR}}$ approximately. If $\omega_{f}$ is of the order of $\omega_{r}^{( \pm)} \sim 1 / \ln (1 / h)$, then Eq. (10) reduces to the following rough asymptotic equation for $\Delta E_{\mathrm{NR}}$ :

$$
\begin{gathered}
\Delta E_{\mathrm{NR}} \sim V_{0}\left(E=E_{s} \pm \Delta E_{\mathrm{NR}}\right) h \ln (1 / h), \\
h \rightarrow 0 .
\end{gathered}
$$

The asymptotic solution of Eq. (11) essentially depends on $V_{0}\left(E_{s} \pm \Delta E_{\mathrm{NR}}\right)$ as a function of $\Delta E_{\mathrm{NR}}$. In this context, all systems can be divided in the following two types.

Type I. The separatrix of the unperturbed system has two or more saddles while the relevant Fourier coefficient $V^{(L)}$ $\equiv V^{(L)}(E, \psi)$ possesses different values on adjacent saddles.
Given that, for $E \rightarrow E_{s}$, the system stays most of time near one of the saddles, the coefficient $V^{(L)}\left(E \rightarrow E_{s}, \psi\right)$ as a function of $\psi$ is nearly a "square wave:" it oscillates between the values at the different saddles. The relevant $K$ is typically odd and, therefore, $V_{0}\left(E \rightarrow E_{s}\right)$ approaches a well-defined nonzero value. Substituting it in Eq. (11), we conclude that

$$
\Delta E_{\mathrm{NR}} \propto h \ln (1 / h), \quad h \rightarrow 0 .
$$

Type II. Either (i) the separatrix of the unperturbed system has a single saddle or (ii) it has more than one saddle but the perturbation coefficient $V^{(L)}$ is identical for all saddles. Then $V^{(L)}\left(E \rightarrow E_{s}, \psi\right)$, as a periodic function of $\psi$, significantly differs from its value at the saddle(s) only during a small part of the period in $\psi$ : this part is $\sim \omega(E) \sim 1 / \ln \left(1 /\left|E_{s}-E\right|\right)$. Hence, $V_{0}\left(E_{s} \pm \Delta E_{\mathrm{NR}}\right) \propto 1 / \ln \left(1 / \Delta E_{\mathrm{NR}}\right)$. Substituting this value in Eq. (11), we conclude that

$$
\Delta E_{\mathrm{NR}} \propto h, \quad h \rightarrow 0 .
$$

Thus, for systems of type I, the maximal width of the SM chaotic layer is proportional to $h$ times a logarithmically large factor $\propto \ln (l / h)$ while, for systems of type II, it is proportional to $h$ times a numerical factor.

As shown below, the variation of energy in between the instants relevant to the $\mathrm{SM}$ is $\sim h$, thus being much less than $\Delta E_{\mathrm{NR}}$ (12) for the systems of type I and being of the same order of magnitude as $\Delta E_{\mathrm{NR}}$ (13) for the systems of type II. Therefore, one may expect that the maximal width of the layer for the original Hamiltonian system $(1), \Delta E^{( \pm)}$, is at least roughly approximated by that for the $\mathrm{SM}, \Delta E_{s m}^{( \pm)}$, so that the above classification of systems is relevant to $\Delta E^{( \pm)}$too. This is confirmed both by the numerical integration of equations of motion of the original Hamiltonian system and by the more accurate theory presented in the next two sections.

\section{ASYMPTOTIC THEORY FOR SYSTEMS OF TYPE I}

For the sake of clarity, we consider a concrete example of type I, while the generalization is straightforward. Let us consider an archetypal example: the ac-driven pendulum (sometimes called as a pendulum subject to a dipole timeperiodic perturbation) $[4,15,16]$

$$
\begin{gathered}
H=H_{0}+h V, \\
H_{0}=\frac{p^{2}}{2}-\cos (q), \quad V=-q \cos \left(\omega_{f} t\right), \quad h \ll 1 .
\end{gathered}
$$

Figure 2 presents the results of computer simulations (i.e., of a numerical integration of the equations of motion) for a few values of $h$ and several values of $\omega_{f}$. It shows that: (1) the function $\Delta E^{(-)}\left(\omega_{f}\right)$ indeed possesses sharp peaks; their heights greatly exceed the estimates by the heuristic [4], adiabatic [13], and mathematical moderate-frequency [9] theories (see the inset); (2) as predicted by the rough estimates in Sec. III, the first peak of $\Delta E^{(-)}\left(\omega_{f}\right) / h$ shifts to smaller values of $\omega_{f}$ while its height grows as $h$ decreases. Below, we develop the leading-order asymptotic theory for $\Delta E^{(-)}\left(\omega_{f}\right)$ [the theory for $\Delta E^{(+)}\left(\omega_{f}\right)$ may be developed analo- 


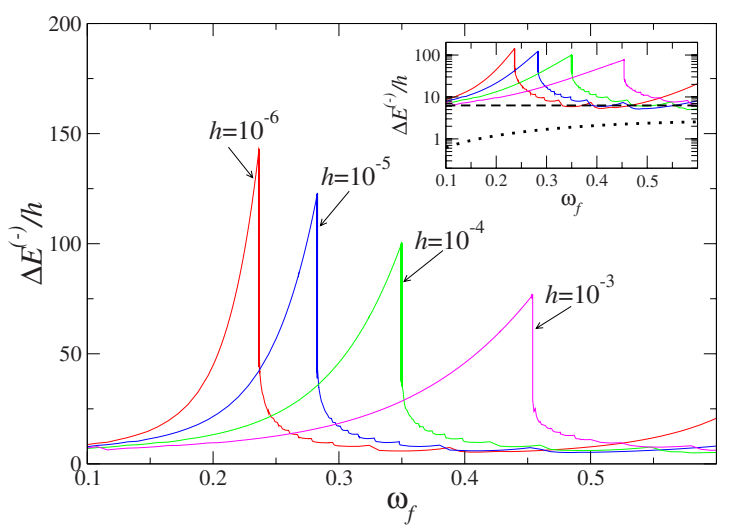

FIG. 2. (Color online) Computer simulations for the ac-driven pendulum (14) (an archetypal example of type I): the deviation $\Delta E^{(-)}$of the lower boundary of the chaotic layer from the separatrix, normalized by the perturbation amplitude $h$, as a function of the perturbation frequency $\omega_{f}$, for various $h$. Inset presents the same data but in logarithmic vertical scale and with the estimates by the heuristic [4], adiabatic [13], and mathematical moderate-frequency [9] theories: the heuristic estimate is shown by the dotted line [32] while the adiabatic and moderate-frequency estimates are shown by the dashed line [33]. Inset explicitly shows that the simulation results exceed the estimates by the former theories by 1 or 2 orders of magnitude for a wide range of frequencies.

gously] and compare it to results of the simulations.

Before moving on, we note that the SM (approximated in the relevant case by the nonlinear resonance dynamics) considers states of the system only at discrete instants. Apart from the variation of energy within the SM dynamics, the variation of energy in the Hamiltonian system occurs also in between the instants relevant to the SM. Given that $\omega_{f} \ll 1$, this latter variation may be considered in adiabatic approximation and it is of the order of $h[13,23]$. As follows from the rough estimates above and from the accurate consideration below, the variation of energy within the SM dynamics for systems of type I is logarithmically larger, i.e., larger by the factor $\ln (1 / h)$. Therefore, the variation of energy in between the instants relevant to the SM may be neglected at the leading-order approximation for systems of type I [the parameter of smallness of the asymptotic theory is $1 / \ln (1 / h)]$ : $\Delta E^{(-)} \simeq \Delta E_{s m}^{(-)}$. For the sake of notational compactness, we shall omit the subscript "sm" further in this section.

For the system (14), the separatrix energy is equal to 1, while the asymptotic (for $E \rightarrow E_{s}-0$ ) dependence $\omega(E)$ is [4]

$$
\begin{aligned}
& \omega(E) \simeq \frac{\pi}{\ln \left[32 /\left(E_{s}-E\right)\right]}, \\
& E_{s}=1, \quad 0<E_{s}-E \ll 1 .
\end{aligned}
$$

Let $\omega_{f}$ be close to one of the odd multiples of $\omega_{r}^{(-)}$. The nonlinear resonance dynamics of the slow variables in the range of the approximately resonant energies may be described as follows [24,34] (cf. also [2-7]):

$$
\frac{d I}{d t}=-\frac{\partial \tilde{H}(I, \tilde{\psi})}{\partial \tilde{\psi}}, \quad \frac{d \tilde{\psi}}{d t}=\frac{\partial \tilde{H}(I, \tilde{\psi})}{\partial I},
$$

$$
\begin{gathered}
\widetilde{H}(I, \widetilde{\psi})=\int_{I\left(E_{s}\right)}^{I} d \widetilde{I}\left(n \omega-\omega_{f}\right)-n h q_{n} \cos (\widetilde{\psi}) \\
\equiv n\left(E-E_{s}\right)-\omega_{f}\left[I-I\left(E_{s}\right)\right]-n h q_{n} \cos (\widetilde{\psi}), \\
I \equiv I(E)=\int_{E_{\min }}^{E} \frac{d \widetilde{E}}{\omega(\widetilde{E})}, \quad E \equiv H_{0}(p, q), \\
\widetilde{\psi}=n \psi-\omega_{f} t, \\
\psi=\pi+\operatorname{sgn}(p) \omega(E) \int_{q_{\min }(E)}^{q} \frac{d \widetilde{q}}{\sqrt{2[E-U(\widetilde{q})]}}+2 \pi l, \\
q_{n} \equiv q_{n}(E)=\frac{1}{2 \pi} \int_{0}^{2 \pi} d \psi q(E, \psi) \cos (n \psi), \\
\left|n \omega-\omega_{f}\right| \ll \omega, \quad n \equiv 2 j-1, \quad j=1,2,3, \ldots,
\end{gathered}
$$

where $I$ and $\psi$ are the canonical variables action and angle, respectively [2-7], $E_{\min }$ is the minimal energy $E \equiv H_{0}(p, q)$ over all $q, p, q_{\min }(E)$ is the minimal coordinate of the conservative motion with a given value of energy $E$, and $l$ is the number of right turning points in the trajectory $[q(\tau)]$ of the conservative motion with energy $E$ and given initial state $\left(q_{0}, p_{0}\right)$.

The resonance Hamiltonian $\widetilde{H}(I, \widetilde{\psi})$ is obtained from the original Hamiltonian $H$ transforming to action-angle variables $I-\psi$, with a further multiplication by $n$, extracting the term $\omega_{f} I$ (that corresponds to the transformation $\psi \rightarrow \tilde{\psi}$ $\left.\equiv n \psi-\omega_{f} t\right)$, and neglecting all the fast-oscillating terms (their effect on the dynamics of slow variables is small: see the estimate of the corrections in Sec. VI below), i.e., keeping only the resonance term in the double Fourier expansion of the perturbation.

Let us derive the asymptotic expression for $I(E)$, substituting the asymptotic expression (15) for $\omega(E)$ into the definition of $I(E)(16)$ and carrying out the integration

$$
I(E) \simeq I\left(E_{s}\right)-\frac{E_{s}-E}{\pi}\left[\ln \left(\frac{32}{E_{s}-E}\right)+1\right] .
$$

As for the asymptotic value $q_{n}\left(E \rightarrow E_{s}-0\right)$, it is easy to see that $q\left(E \rightarrow E_{s}-0, \psi\right)$, as a function of $\psi$, asymptotically approaches a "square wave," oscillating between $-\pi$ and $\pi$, so that, for sufficiently small $j$,

$$
\begin{gathered}
q_{2 j-1}\left(E \rightarrow E_{s}-0\right) \simeq(-1)^{j+1} \frac{2}{2 j-1}, \\
q_{2 j}=0, \\
j=1,2, \ldots \ll \frac{\pi}{2 \omega(E)} .
\end{gathered}
$$

The next issue is the analysis of the phase space of the resonant Hamiltonian (16). Substituting $\widetilde{H}$ (16) into the equa- 
tions of motion (16), it is easy to see that their stationary points have the following values of the slow angle:

$$
\tilde{\psi}_{+}=\pi, \quad \tilde{\psi}_{-}=0
$$

while the corresponding action is determined by the equation

$$
n \omega-\omega_{f} \mp n h \frac{d q_{n}}{d I}=0, \quad n \equiv 2 j-1
$$

where the sign "₹"

As usual (cf. [2-7,24,34]), the term $\propto h$ in Eq. (20) may be neglected in the leading-order approximation and Eq. (20) reduces to the resonance condition

$$
(2 j-1) \omega\left(E_{r}^{(j)}\right)=\omega_{f},
$$

the lowest-order solution of which is

$$
E_{s}-E_{r}^{(j)} \simeq 32 \exp \left(-\frac{(2 j-1) \pi}{\omega_{f}}\right) .
$$

Equations (19) and (22) together with Eq. (17) explicitly determine the elliptic and hyperbolic points of the Hamiltonian (16). The hyperbolic point is often called "saddle" and corresponds to $\tilde{\psi}_{+}$or $\tilde{\psi}_{-}$in Eq. (19) for even or odd $j$, respectively. The saddle point generates the resonance separatrix. Using the asymptotic relations (17) and (18) together with Eqs. (19) and (22), we obtain that the resonance Hamiltonian (16) takes the following asymptotic value in the saddle:

$$
\tilde{H}_{\text {saddle }} \simeq \frac{E_{s}-E_{r}^{(j)}}{\pi} \omega_{f}-2 h \simeq \frac{\omega_{f}}{\pi} 32 \exp \left(-\frac{\pi(2 j-1)}{\omega_{f}}\right)-2 h .
$$

As explained in Sec. II above, $\Delta E^{(-)}\left(\omega_{f}\right)$ possesses a local maximum at $\omega_{f}$ for which the resonance separatrix is tangent to the lower GSS curve [Fig. 1(a)]. For the relevant frequency range $\omega_{f} \rightarrow 0$, the separatrix split (which represents the maximum deviation of the energy along the GSS curve from $E_{s}$ ) approaches the following value [4] in the asymptotic limit $h \rightarrow 0$,

$$
\delta \simeq 2 \pi h, \quad \omega_{f} \ll 1 .
$$

As it is shown further down, the variation of energy along the relevant resonance trajectories is much larger. Therefore, in the leading-order approximation, the GSS curve may be simply replaced by the separatrix of the unperturbed system, i.e., by the horizontal line $E=E_{s}$ or, equivalently, $I=I\left(E_{s}\right)$. Then the tangency occurs at $\widetilde{\psi}$ shifted from the saddle by $\pi$ so that the condition of tangency is written as

$$
\tilde{H}_{\text {saddle }}=\tilde{H}\left[I=I\left(E_{s}\right), \quad \tilde{\psi}=\tilde{\psi}_{\text {saddle }}+\pi\right] \equiv 2 h .
$$

Substituting here $\tilde{H}_{\text {saddle }}(23)$, we finally obtain the following transcendental equation for $\omega_{f}=\omega_{\max }^{(j)}$ :
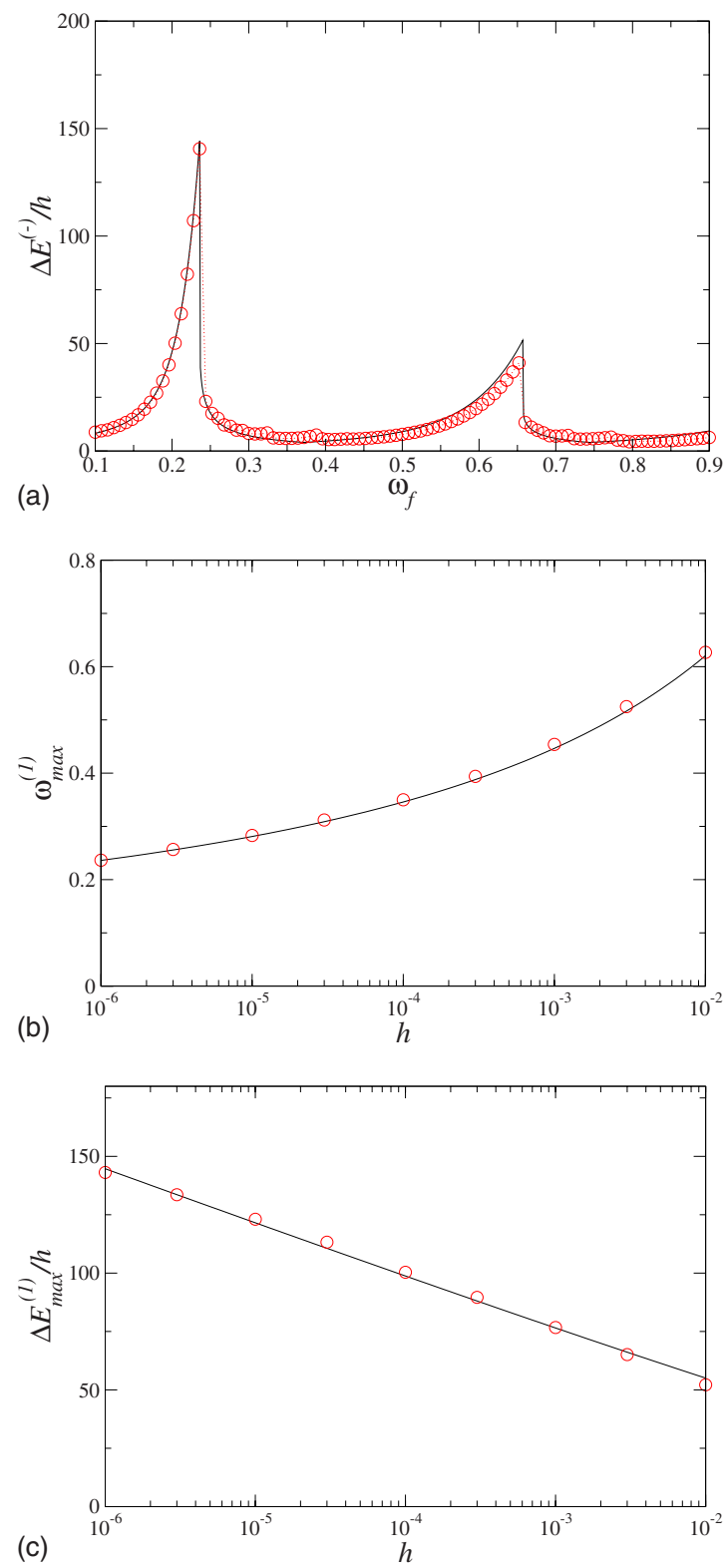

FIG. 3. (Color online) An archetypal example of type I: acdriven pendulum (14). Comparison of theory (solid lines) and simulations (circles): (a) the deviation $\Delta E^{(-)}\left(\omega_{f}\right)$ of the lower boundary of the chaotic layer from the separatrix, normalized by the perturbation amplitude $h$, as a function of the perturbation frequency $\omega_{f}$, for $h=10^{-6}$; the theory is by Eqs. (26), (31), (32), (38), (39), and (41). (b) The frequency of the first maximum in $\Delta E^{(-)}\left(\omega_{f}\right)$ as a function of $h$; the theory is by Eq. (26). (c) The first maximum in $\Delta E^{(-)}\left(\omega_{f}\right) / h$ as a function of $h$; the theory is by Eqs. (34) and (26).

$$
x \exp (x)=\frac{8(2 j-1)}{h}, \quad x \equiv \frac{(2 j-1) \pi}{\omega_{\max }^{(j)}} .
$$

Figure 3(b) demonstrates the excellent agreement between Eq. (26) and the results of simulations for the Hamiltonian system in a wide range of $h$.

In the asymptotic limit $h \rightarrow 0$, the lowest-order explicit solution of Eq. (26) is 


$$
\omega_{\max }^{(j)} \simeq \frac{(2 j-1) \pi}{\ln \left(\frac{8(2 j-1)}{h}\right)}, \quad j=1,2, \ldots \ll \ln \left(\frac{1}{h}\right) .
$$

As follows from Eq. (26), the value of $E_{s}-E_{r}^{(j)}(22)$ for $\omega_{f}$ $=\omega_{\max }^{(j)}$ is

$$
E_{s}-E_{r}^{(j)}\left(\omega_{f}=\omega_{\max }^{(j)}\right)=\frac{4 \pi h}{\omega_{\max }^{(j)}} .
$$

Its leading-order expression is

$$
\begin{aligned}
E_{s}-E_{r}^{(j)}\left(\omega_{f}=\omega_{\max }^{(j)}\right) & \simeq \frac{4 h}{2 j-1} \ln \left(\frac{8(2 j-1)}{h}\right), \\
h & \rightarrow 0 .
\end{aligned}
$$

If $\omega_{f} \leq \omega_{\max }^{(j)}$, then, in the chaotic layer, the largest deviation of energy from the separatrix value corresponds to the minimum energy $E_{\min }^{(j)}$ on the nonlinear resonance separatrix [Figs. 1(a) and 1(b)], which occurs at $\widetilde{\psi}$ shifted by $\pi$ from the saddle. The condition of equality of $\widetilde{H}$ at the saddle and at the minimum of the resonance separatrix is written as

$$
\tilde{H}_{\text {saddle }}=\tilde{H}\left[I\left(E_{\text {min }}^{(j)}\right), \tilde{\psi}_{\text {saddle }}+\pi\right] .
$$

Let us seek its asymptotic solution in the form

$$
\begin{gathered}
E_{s}-E_{\min }^{(j)} \equiv \Delta E_{l}^{(j)}=(1+y)\left(E_{s}-E_{r}^{(j)}\right) \\
\simeq(1+y) 32 \exp \left[-\frac{\pi(2 j-1)}{\omega_{f}}\right], \\
y \gtrsim 1 .
\end{gathered}
$$

Substituting Eqs. (31) and (23) into Eq. (30) and calculating the right-hand part by means of Eqs. (16)-(19) and (22), we obtain for $y$ the following transcendental equation:

$$
\begin{gathered}
(1+y) \ln (1+y)-y=\frac{h}{8(2 j-1)} x_{f} \exp \left(x_{f}\right), \\
x_{f} \equiv \frac{\pi(2 j-1)}{\omega_{f}}, \quad \omega_{f} \leq \omega_{\max }^{(j)}, \quad y>0,
\end{gathered}
$$

where $\omega_{\max }^{(j)}$ is given by Eq. (26).

Equations (31) and (32) describe the left wing of the $j$ th peak of $\Delta E^{(-)}\left(\omega_{f}\right)$. Figure $3(\mathrm{a})$ demonstrates the good agreement between our analytic theory and simulations for the Hamiltonian system.

As follows from Eq. (26), Eq. (32) for $\omega_{f}=\omega_{\max }^{(j)}$ reduces to the relation $\ln (1+y)=1$, i.e.,

$$
1+y\left(\omega_{\max }^{(j)}\right)=e .
$$

As follows from Eqs. (33), (31), and (28), the maximum for a given peak is

$$
\Delta E_{\max }^{(j)} \equiv E_{s}-E_{\min }^{(j)}\left(\omega_{\max }^{(j)}\right)=\frac{4 \pi e h}{\omega_{\max }^{(j)}} .
$$

Figure 3(c) shows the excellent agreement of this expression with the results of simulations for the Hamiltonian system in a wide range of $h$.

The leading-order expression for $\Delta E_{\max }^{(j)}$ is

$$
\Delta E_{\max }^{(j)} \simeq \frac{4 e h}{2 j-1} \ln [8(2 j-1) / h], \quad h \rightarrow 0,
$$

which confirms the rough estimate (12).

As $\omega_{f}$ decreases, $y$ increases exponentially sharply, as follows from Eq. (32). In order to understand how $\Delta E_{l}^{(j)}$ decreases upon decreasing $\omega_{f}$, it is convenient to rewrite Eq. (31) expressing the exponent by means of Eq. (32),

$$
\Delta E_{l}^{(j)}\left(\omega_{f}\right)=\frac{4 \pi h}{\omega_{f}[\ln (1+y)-y /(1+y)]} .
$$

It follows from Eqs. (32) and (36) that $\Delta E_{l}^{(j)}$ decreases powerlike rather than exponentially when $\omega_{f}$ is decreased. In particular, $\Delta E_{l}^{(j)} \propto 1 /\left(\omega_{\max }^{(j)}-\omega_{f}\right)$ for the far part of the wing.

As for the right wing of the peak, i.e., for $\omega_{f}>\omega_{\max }^{(j)}$, over the chaotic layer, the largest deviation of energy from the separatrix value corresponds to the minimum of the resonance trajectory tangent to the GSS curve [Fig. 1(c)]. The value of $\tilde{\psi}$ in the minimum coincides with $\widetilde{\psi}_{\text {saddle }}$ In the leading-order approximation, the GSS curve may be replaced by the horizontal line $I=I\left(E_{s}\right)$, so that the tangency occurs at $\widetilde{\psi}=\widetilde{\psi}_{\text {saddle }}+\pi$. Then the energy at the minimum $E_{\text {min }}^{(j)}$ can be found from the equation

$$
\tilde{H}\left[I\left(E_{s}\right), \tilde{\psi}_{\text {saddle }}+\pi\right]=\tilde{H}\left[I\left(E_{\text {min }}^{(j)}\right), \tilde{\psi}_{\text {saddle }}\right] .
$$

Let us seek its asymptotic solution in the form

$$
\begin{gathered}
E_{s}-E_{\min }^{(j)} \equiv \Delta E_{r}^{(j)}= \\
z\left(E_{s}-E_{r}^{(j)}\right) \simeq z 32 \exp \left(-\frac{\pi(2 j-1)}{\omega_{f}}\right), \\
0<z<1, \quad z \sim 1 .
\end{gathered}
$$

Substituting Eq. (38) into Eq. (37) and calculating the righthand part by means of Eqs. (16)-(19) and (22), we obtain for $z$ the following transcendental equation:

$$
\begin{gathered}
z[1+\ln (1 / z)]=\frac{h}{8(2 j-1)} x_{f} \exp \left(x_{f}\right), \\
x_{f} \equiv \frac{\pi(2 j-1)}{\omega_{f}}, \quad \omega_{f}>\omega_{\max }^{(j)}, \quad 0<z<1,
\end{gathered}
$$

where $\omega_{\max }^{(j)}$ is given by Eq. (26).

Equations (38) and (39) describe the right wing of the $j$ th peak of $\Delta E^{(-)}\left(\omega_{f}\right)$. Figure 3(a) shows the good agreement between our analytic theory and simulations.

As follows from Eq. (26), the solution of Eq. (39) for $\omega_{f} \rightarrow \omega_{\max }^{(j)}$ is $z \rightarrow 1$, so the right wing starts from the value given by Eq. (28) [or, approximately, by Eq. (29)].

Expressing the exponent in Eq. (38) from Eq. (39), we obtain the following equation: 


$$
\Delta E_{r}^{(j)}\left(\omega_{f}\right)=\frac{4 \pi h}{\omega_{f}[1+\ln (1 / z)]} .
$$

It follows from Eqs. (39) and (40) that $\Delta E_{r}^{(j)}$ decreases powerlike rather than exponentially for increasing $\omega_{f}$. In particular, $\Delta E_{r}^{(j)} \propto 1 /\left(\omega_{f}-\omega_{\max }^{(j)}\right)$ in the far part of the wing. The further analysis of the asymptotic shape of the peak is done in Sec. VII below.

Beyond the peaks, the function $\Delta E^{(-)}\left(\omega_{f}\right)$ is logarithmically small in comparison to the maxima of the peaks. The functions $\Delta E_{l}^{(j)}\left(\omega_{f}\right)$ and $\Delta E_{r}^{(j)}\left(\omega_{f}\right)$ in the ranges beyond the peaks are also logarithmically small. Hence, nearly any combination of the functions $\Delta E_{r}^{(j)}\left(\omega_{f}\right)$ and $\Delta E_{l}^{(j+1)}\left(\omega_{f}\right)$ which is close to $\Delta E_{r}^{(j)}\left(\omega_{f}\right)$ in the vicinity of $\omega_{\max }^{(j)}$ and to $\Delta E_{l}^{(j+1)}\left(\omega_{f}\right)$ in the vicinity of $\omega_{\max }^{(j+1)}$ may be considered as an approximation of the function $\Delta E^{(-)}\left(\omega_{f}\right)$ with a logarithmic accuracy with respect to the maxima of the peaks, $\Delta E_{\max }^{(j)}$ and $\Delta E_{\max }^{(j+1)}$, in the whole range $\left[\omega_{\max }^{(j)}, \omega_{\max }^{(j+1)}\right]$. One of the easiest combinations is the following:

$$
\begin{gathered}
\Delta E^{(-)}\left(\omega_{f}\right)=\Delta E_{l}^{(1)}\left(\omega_{f}\right) \quad \text { for } \quad \omega_{f}<\omega_{\max }^{(1)}, \\
\Delta E^{(-)}\left(\omega_{f}\right)=\max \left\{\Delta E_{r}^{(j)}\left(\omega_{f}\right), \Delta E_{l}^{(j+1)}\left(\omega_{f}\right)\right\} \quad \text { for } \omega_{\max }^{(j)}<\omega_{f} \\
<\omega_{\max }^{(j+1)}, \\
\quad j=1,2, \ldots \ll \frac{\pi}{2 \omega_{\max }^{(1)}} .
\end{gathered}
$$

We used this function in Fig. 3(a) and the analogous combination will be also used in the other cases.

In fact, the theory may be generalized in such a way that Eq. (41) would well approximate $\Delta E^{(-)}\left(\omega_{f}\right)$ in the ranges far beyond the peaks with a logarithmic accuracy even with respect to $\Delta E^{(-)}\left(\omega_{f}\right)$ itself rather than to $\Delta E_{\max }^{(j)}$ only (cf. the next section). However, we do not do this in the present case, being interested primarily in the leading-order description of the peaks. Finally, we demonstrate in Fig. 4 that the lowestorder theory describes quite well the layer boundaries even in the Poincaré section rather than only in energy or action.

\section{ASYMPTOTIC THEORY FOR SYSTEMS OF TYPE II}

We shall consider two characteristic examples of type II corresponding to the classification given in Sec. III. As an example of the system where the separatrix of the unperturbed system possesses a single saddle, we shall consider the ac-driven Duffing oscillator [7-9,20]. As an example of the system where the separatrix possesses more than one saddle while the perturbation takes equal values at the saddles, we shall consider the pendulum with an oscillating suspension point $[7-9,18,23]$. The treatment of these cases is similar in many respects to the one presented in Sec. IV above. So, we present it in less details, emphasizing the differences.

\section{A. ac-driven Duffing oscillator}

Consider the following archetypal Hamiltonian [7-9,20]:

$$
H=H_{0}+h V \text {, }
$$

$$
H_{0}=\frac{p^{2}}{2}-\frac{q^{2}}{2}+\frac{q^{4}}{4}, \quad V=-q \cos \left(\omega_{f} t\right), \quad h \ll 1 .
$$

The asymptotic dependence of $\omega(E)$ on $E$ for $E$ below the separatrix energy $E_{s}=0$ is the following $[7,35]$ :

$$
\begin{aligned}
& \omega(E) \simeq \frac{2 \pi}{\ln \left[16 /\left(E_{s}-E\right)\right]}, \\
& E_{s}=0, \quad 0<E_{s}-E \ll 1 .
\end{aligned}
$$

Correspondingly, the resonance values of energies [determined by the condition analogous to Eq. (21)] are

$$
E_{s}-E_{r}^{(j)}=16 \exp \left(-\frac{2 \pi j}{\omega_{f}}\right), \quad j=1,2,3, \ldots .
$$

The asymptotic dependence of $I(E)$ is

$$
I(E) \simeq I\left(E_{s}\right)-\frac{E_{s}-E}{2 \pi}\left[\ln \left(\frac{16}{E_{s}-E}\right)+1\right] .
$$

The nonlinear resonance dynamics is described by the resonance Hamiltonian $\tilde{H}$ which is identical to Eq. (16) in form. Obviously, the actual dependencies $\omega(E)$ and $I(E)$ are given by Eqs. (43) and (45), respectively. The most important difference is in $q_{j}(E)$ : instead of a nonzero value [see Eq. (18)], it approaches 0 as $E \rightarrow E_{s}$. Namely, it is $\propto \omega(E)[7,35]$,

$$
\begin{gathered}
q_{j}(E) \simeq \frac{1}{\sqrt{2}} \omega(E), \quad j=1,2, \ldots \ll \frac{\pi}{\omega(E)}, \\
E \rightarrow E_{s}-0,
\end{gathered}
$$

i.e., $q_{j}$ is much smaller than in systems of type I [cf. Eq. (18)]. Due to this, the resonance is "weaker." At the same time, the separatrix split $\delta$ is also smaller, namely, $\sim h \omega_{f}$ (cf. [24]) rather than $\sim h$ as for the systems of type I. That is why the separatrix chaotic layer is still dominated by the resonance dynamics while the matching of the separatrix map and nonlinear resonance dynamics is still valid in the asymptotic limit $h \rightarrow 0$ [24].

Similarly to the previous section, we find the value of $\tilde{H}$ in the saddle in the leading-order approximation [36]

$$
\tilde{H}_{\text {saddle }} \simeq \omega_{f}\left(\frac{E_{s}-E_{r}^{(j)}}{2 \pi}-\frac{h}{\sqrt{2}}\right),
$$

where $E_{s}-E_{r}^{(j)}$ is given in Eq. (44). As before, the maximum width of the layer corresponds to $\omega_{f}$, for which the resonance separatrix is tangent to the GSS curve [Fig. 1(a)]. It can be shown [24] that the angle of tangency asymptotically approaches $\tilde{\psi}_{\text {saddle }}+\pi=\pi$ while the energy still lies in the resonance range, where $\omega(E) \approx \omega_{r}^{(-)} \approx \omega_{f} / j$. Using the expressions for $\widetilde{H}(E, \widetilde{\psi})$ [cf. Eq. (16)], $I(E)$ [Eq. (45)], and $q_{j}(E)$ [Eq. (46)] and taking into account that in the tangency $E$ $<\delta \sim h \omega_{f} \ll h$, the value of $\widetilde{H}$ at the tangency reads in the leading-order approximation as

$$
\tilde{H}_{\text {tangency }} \simeq \omega_{f} \frac{h}{\sqrt{2}} .
$$


Allowing for Eqs. (47) and (48), the condition for the maximum, $\widetilde{H}_{\text {saddle }}=\widetilde{H}_{\text {tangency }}$, reduces to

$$
E_{s}-E_{r}^{(j)}\left(\omega_{\max }^{(j)}\right) \simeq 2 \pi \sqrt{2} h .
$$

Thus, these values $E_{s}-E_{r}^{(j)}$ are logarithmically smaller than the corresponding values (28) for systems of type I.

The values of $\omega_{f}$ corresponding to the maxima of the peaks in $\Delta E^{(-)}\left(\omega_{f}\right)$ are readily obtained from Eqs. (49) and (44):

$$
\omega_{\max }^{(j)} \simeq \frac{2 \pi j}{\ln [4 \sqrt{2} /(\pi h)]}, \quad j=1,2, \ldots \ll \ln (1 / h) .
$$

The derivation of the shape of the peaks for the chaotic layer of the separatrix map in the leading order, i.e., within the NR approximation, is similar to that for type I. So, we present only the results, marking them with the subscript "NR."

The left wing of the $j$ th peak of $\Delta E_{\mathrm{NR}}^{(-)}\left(\omega_{f}\right)$ is described by the function

$$
\begin{aligned}
\Delta E_{l, \mathrm{NR}}^{(j)}\left(\omega_{f}\right) & =16(1+y) \exp \left(-\frac{2 \pi j}{\omega_{f}}\right) \\
& \equiv \frac{2 \pi \sqrt{2} h}{\ln (1+y)-y /(1+y)}, \quad \omega_{f} \leq \omega_{\max }^{(j)},
\end{aligned}
$$

where $y$ is the positive solution of the transcendental equation

$$
(1+y) \ln (1+y)-y=\frac{\pi h}{4 \sqrt{2}} \exp \left(\frac{2 \pi j}{\omega_{f}}\right), \quad y>0 .
$$

Similarly to the type I case, $1+y\left(\omega_{\max }^{(j)}\right)=e$, so that

$$
\Delta E_{\max , \mathrm{NR}}^{(j)}=e\left[E_{s}-E_{r}^{(j)}\left(\omega_{\max }^{(j)}\right)\right] \simeq 2 \pi e \sqrt{2} h .
$$

Equation (53) confirms the rough estimate Eq. (13).

The right wing of the peak is described by the function

$$
\Delta E_{r, \mathrm{NR}}^{(j)}\left(\omega_{f}\right)=16 z \exp \left(-\frac{2 \pi j}{\omega_{f}}\right) \equiv \frac{2 \pi \sqrt{2} h}{1+\ln (1 / z)}, \quad \omega_{f}>\omega_{\max }^{(j)},
$$

where $z<1$ is the solution of the transcendental equation

$$
z[1+\ln (1 / z)]=\frac{\pi h}{4 \sqrt{2}} \exp \left(\frac{2 \pi j}{\omega_{f}}\right), \quad 0<z<1 .
$$

Similarly to the type I case, $z\left(\omega_{f} \rightarrow \omega_{\max }^{(j)}\right) \rightarrow 1$.

As follows from Eqs. (49) and (53), the typical variation of energy within the nonlinear resonance dynamics (that approximates the separatrix map dynamics) is $\propto h$. For the Hamiltonian system, the variation of energy in between the discrete instants corresponding to the separatrix map $[4-7,24,26]$ is also $\propto h$. Therefore, unlike the case of type I, one needs to take it into account even at the leading-order approximation. Let us consider the right well of the Duffing potential (the results for the left well are identical) and denote by $t_{k}$ the instant at which the energy $E$ at a given $k$ th step of the separatrix map is taken: it corresponds to the beginning of the $k$ th pulse of velocity $[4,24]$, i.e., the corresponding $q$ is close to a left turning point $q_{l t p}$ in the trajectory
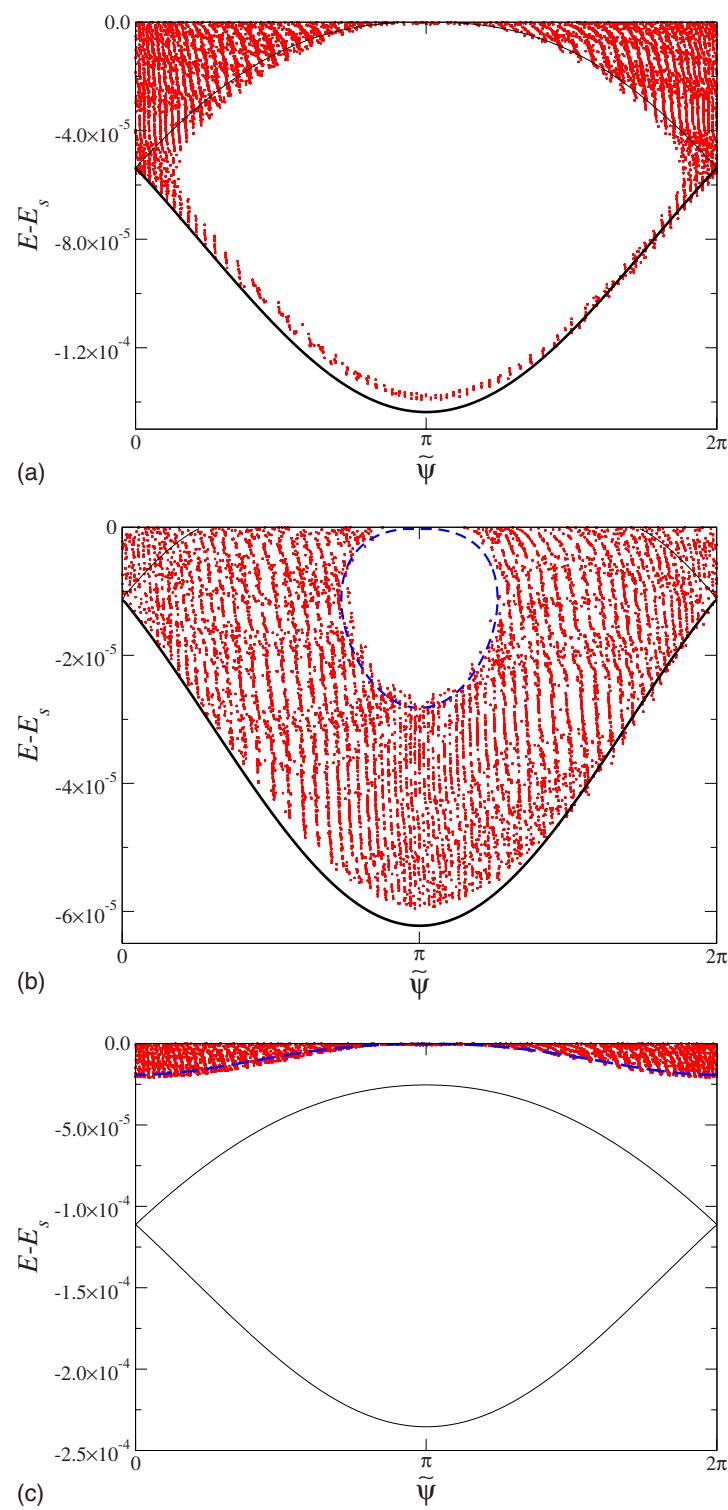

FIG. 4. (Color online) A few characteristic Poincaré sections in the $2 \pi$ interval of the energy-angle plane for the system (14) with $h=10^{-6}$ and $\omega_{f}$ equal to (a) 0.236 (maximum), (b) 0.21 (left wing), and (c) 0.25 (right wing). Results of the numerical integration of the equations of motion for the original Hamiltonian (14) are shown by red dots. The NR separatrix calculated in the leading-order approximation [i.e., by the integration of the resonant equations of motion (16) in which $\omega(E), I(E)$, and $q_{1}(E)$ are approximated by the explicit formulas (15), (17), and (18), respectively] is drawn by the black solid line. The NR trajectory (calculated in the leading-order approximation) tangent to the line $E=E_{s}$ is drawn by the blue dashed line. The outer boundary (marked by a thicker line) is approximated by the lower part of the NR separatrix in the cases (a) and (b) and by the tangent NR trajectory in the case (c). The boundary of the island of stability in the cases (a) and (b) is approximated by the tangent NR trajectory [which coincides in the case (a) with the NR separatrix].

$[q(\tau)]$. Let us also take into account that the relevant frequencies are small so that the adiabatic approximation may be used. Thus, the change of energy from $t_{k}$ up to a given 

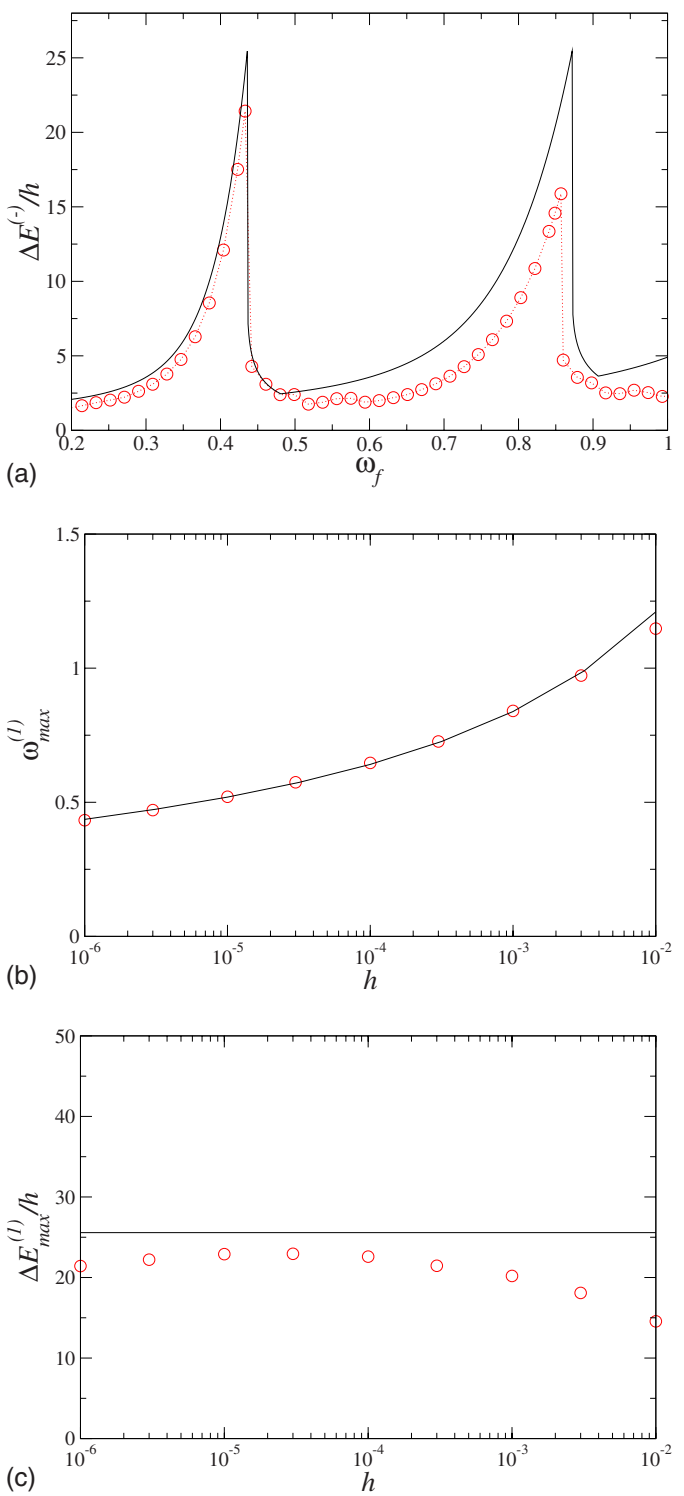

FIG. 5. (Color online) An archetypal example of type II: acdriven Duffing oscillator (42). Comparison of theory (solid lines) and simulations (circles). (a) The deviation $\Delta E^{(-)}\left(\omega_{f}\right)$ of the lower boundary of the chaotic layer from the separatrix, normalized by the perturbation amplitude $h$, as a function of the perturbation frequency $\omega_{f}$, for $h=10^{-6}$; the theory is by Eqs. (41), (50)-(52), (54), (55), (58), and (60). (b) The frequency of the first maximum in $\Delta E^{(-)}\left(\omega_{f}\right)$ as a function of $h$; the theory is by Eq. (50). (c) The first maximum in $\Delta E^{(-)}\left(\omega_{f}\right) / h$ as a function of $h$; the theory is by Eq. (59).

instant $t$ during the following pulse of velocity $\left(t-t_{k} \sim 1\right)$ may be calculated as

$$
\begin{aligned}
\Delta E=\int_{t_{k}}^{t} d \tau \dot{q} h \cos \left(\omega_{f} \tau\right) & \simeq h \cos \left(\omega_{f} t_{k}\right) \int_{t_{k}}^{t} d \tau \dot{q} \\
& =h \cos \left(\omega_{f} t_{k}\right)\left(q(t)-q_{l t p}\right)
\end{aligned}
$$

For the motion near the separatrix, the velocity pulse corresponds approximately to $\psi=0$ [see the definition of $\psi \mathrm{Eq}$.
(16)]. Thus, the corresponding slow angle is $\widetilde{\psi} \equiv j \psi-\omega_{f} t_{k} \simeq$ $-\omega_{f} t_{k}$.

For the left wing of the peak of $\Delta E^{(-)}\left(\omega_{f}\right)$ (including the maximum of the peak too), the boundary of the chaotic layer of the separatrix map is formed by the lower part of the NR separatrix [Figs. 1(a) and 1(b)]. The minimum energy along this separatrix occurs at $\widetilde{\psi}=\pi$. Taking this into account and also that $\tilde{\psi} \simeq-\omega_{f} t_{k}$, we conclude that $\cos \left(\omega_{f} t_{k}\right) \simeq-1$. So, $\Delta E \leq 0$, i.e., it does lower the minimum energy of the layer of the Hamiltonian system. The maximum lowering occurs at the right turning point $q_{r t p}$,

$$
\max (|\Delta E|) \simeq h\left(q_{r t p}-q_{l t p}\right)=\sqrt{2} h .
$$

We conclude that the left wing of the $j$ th peak is described by the following formula:

$$
\Delta E_{l}^{(j)}\left(\omega_{f}\right) \simeq \Delta E_{l, \mathrm{NR}}^{(j)}\left(\omega_{f}\right)+\sqrt{2} h, \quad \omega_{f} \leq \omega_{\max }^{(j)},
$$

where $\Delta E_{l, \mathrm{NR}}^{(j)}\left(\omega_{f}\right)$ is given by Eqs. (51) and (52). In particular, the maximum of the peak is

$$
\Delta E_{\max }^{(j)} \simeq(2 \pi e+1) \sqrt{2} h \approx 25.6 h .
$$

For the right wing of the peak, the minimum energy of the layer of the separatrix map occurs at $\tilde{\psi}$ coinciding with $\tilde{\psi}_{\text {saddle }}$ [Fig. 1(c)], i.e., equal to 0 . As a result, $\cos \left(\omega_{f} t_{k}\right) \simeq 1$ and, hence, $\Delta E \geq 0$. So, this variation cannot lower the minimal energy of the layer for the main part of the wing, i.e., for $\omega_{f} \leq \omega_{\text {bend }}^{(j)}$ where $\omega_{\text {bend }}^{(j)}$ is defined by the condition $\Delta E_{r, \mathrm{NR}}^{(j)}$ $=\max (|\Delta E|) \equiv \sqrt{2} h$. For $\omega_{f}>\omega_{\text {bend }}^{(j)}$, the minimal energy in the layer occurs at $\tilde{\psi}=\pi$ and it is determined exclusively by the variation of energy during the velocity pulse (the NR contribution is close to zero at such $\widetilde{\psi}$ ). Thus, we conclude that there is a bending of the wing at $\omega_{f}=\omega_{\text {bend }}^{(j)}$,

$$
\begin{gathered}
\Delta E_{r}^{(j)}\left(\omega_{f}\right)=\Delta E_{r, \mathrm{NR}}^{(j)}\left(\omega_{f}\right), \quad \omega_{\max }^{(j)}<\omega_{f} \leq \omega_{\text {bend }}^{(j)}, \\
\Delta E_{r}^{(j)}\left(\omega_{f}\right)=\sqrt{2} h, \quad \omega_{f} \geq \omega_{\text {bend }}^{(j)}, \\
\omega_{\text {bend }}^{(j)}=\frac{2 \pi j}{\ln (8 \sqrt{2} / h)+1-2 \pi},
\end{gathered}
$$

where $\Delta E_{r, \mathrm{NR}}^{(j)}\left(\omega_{f}\right)$ is given by Eqs. (54) and (55).

Analogously to the previous case, $\Delta E^{(-)}\left(\omega_{f}\right)$ may be approximated in the whole frequency range by Eq. (41) with $\Delta E_{l}^{(j)}$ and $\Delta E_{r}^{(j)}$ given by Eqs. (58) and (60), respectively. Moreover, unlike the previous case, now the theory accurately describes also the range far beyond the peaks: $\Delta E^{(-)}$is dominated in this range by the velocity pulse contribution $\Delta E$, which is accurately taken into account both by Eqs. (58) and (60). Figure 5 shows a very reasonable agreement between theory and simulations, especially for the first peak [37].

\section{B. Pendulum with an oscillating suspension point}

Consider the archetypal Hamiltonian [7-9,18,23]

$$
H=H_{0}+h V,
$$




$$
\begin{gathered}
H_{0}=\frac{p^{2}}{2}+\cos (q), \quad V=-\cos (q) \cos \left(\omega_{f} t\right), \\
h \ll 1 .
\end{gathered}
$$

Though the treatment is similar to the previous case, there are also characteristic differences. One of them is the following: although the resonance Hamiltonian is similar to the Hamiltonian (16), instead of the Fourier component of the coordinate, $q_{n}$, there should be the Fourier component of $\cos (q)$, which we shall denote as $V_{n}$. It can be shown to read as

$$
\begin{gathered}
V_{2 j} \simeq(-1)^{j+1} \frac{4}{\pi} \omega(E), \quad 0<E_{s}-E \ll 1, \\
V_{2 j-1}=0, \\
j=1,2, \ldots \ll \frac{2 \pi}{\omega(E)}, \\
V_{n} \equiv \frac{1}{2 \pi} \int_{0}^{2 \pi} d \psi \cos (q) \cos (n \psi) .
\end{gathered}
$$

The description of the chaotic layer of the separatrix map at the lowest order, i.e., within the NR approximation, is similar to that for the ac-driven Duffing oscillator. So, we present only the results, marking them with the subscript NR.

The frequency of the maximum of a given $j$ th peak is

$$
\omega_{\max }^{(j)} \simeq \frac{2 \pi j}{\ln (4 / h)}, \quad j=1,2, \ldots \ll \ln (4 / h) .
$$

This expression well agrees with simulations for the Hamiltonian system [Fig. 6(b)]. To logarithmic accuracy, Eq. (63) coincides with the formula following from Eq. (8) of [18] or from Eq. (21) of [23] taken in the asymptotic limit $h \rightarrow 0$ (or, equivalently, $\omega_{\max }^{(j)} \rightarrow 0$ ).

The left wing of the $j$ th peak of $\Delta E_{\mathrm{NR}}^{(-)}\left(\omega_{f}\right)$ is described by the function

$$
\begin{aligned}
\Delta E_{l, \mathrm{NR}}^{(j)}\left(\omega_{f}\right) & =32(1+y) \exp \left(-\frac{2 \pi j}{\omega_{f}}\right) \\
& \equiv \frac{8 h}{\ln (1+y)-y /(1+y)}, \quad \omega_{f} \leq \omega_{\max }^{(j)},
\end{aligned}
$$

where $y$ is the positive solution of the transcendental equation

$$
(1+y) \ln (1+y)-y=\frac{h}{4} \exp \left(\frac{2 \pi j}{\omega_{f}}\right), \quad y>0 .
$$

Similarly to the previous cases, $1+y\left(\omega_{\max }^{(j)}\right)=e$. Hence,

$$
\Delta E_{\max , \mathrm{NR}}^{(j)}=e\left[E_{s}-E_{r}^{(j)}\left(\omega_{\max }^{(j)}\right)\right]=8 e h .
$$

Equation (66) confirms the rough estimate (13).

The right wing of the peak is described by the function
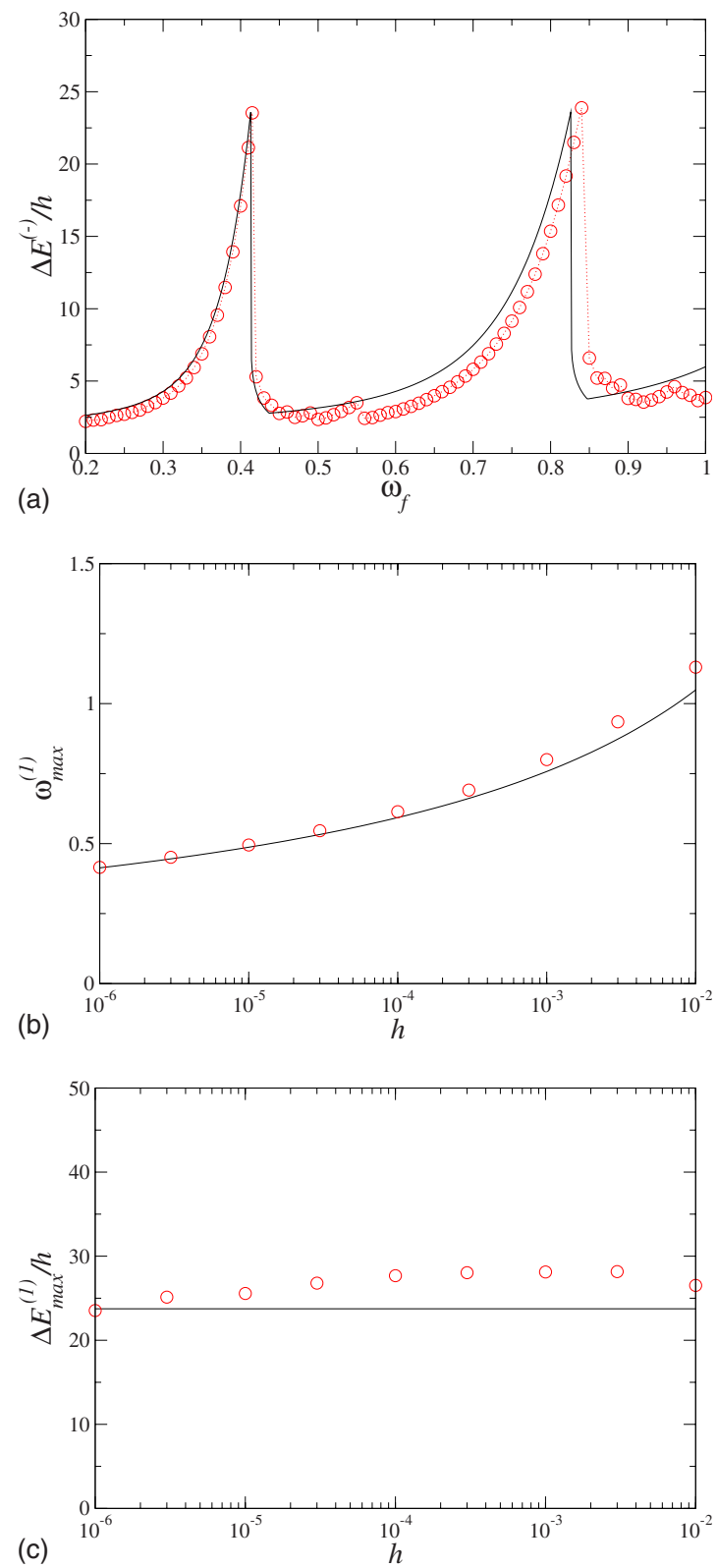

FIG. 6. (Color online) An archetypal example of type II: pendulum with an oscillating suspension point (61). Comparison of theory (solid lines) and simulations (circles). (a) The deviation $\Delta E^{(-)}\left(\omega_{f}\right)$ of the lower boundary of the chaotic layer from the separatrix, normalized by the perturbation amplitude $h$, as a function of the perturbation frequency $\omega_{f}$, for $h=10^{-6}$; the theory is by Eqs. (41), (63)-(65), (67), (68), (71), and (73). (b) The frequency of the first maximum in $\Delta E^{(-)}\left(\omega_{f}\right)$ as a function of $h$; the theory is by Eq. (63). (c) The first maximum in $\Delta E^{(-)}\left(\omega_{f}\right) / h$ as a function of $h$; the theory is by Eq. (72).

$$
\Delta E_{r, \mathrm{NR}}^{(j)}\left(\omega_{f}\right)=32 z \exp \left(-\frac{2 \pi j}{\omega_{f}}\right) \equiv \frac{8 h}{1+\ln (1 / z)}, \quad \omega_{f}>\omega_{\max }^{(j)},
$$




$$
z[1+\ln (1 / z)]=\frac{h}{4} \exp \left(\frac{2 \pi j}{\omega_{f}}\right), \quad 0<z<1 .
$$

Similarly to the previous cases, $z\left(\omega_{f} \rightarrow \omega_{\max }^{(j)}\right) \rightarrow 1$.

Consider now the variation of energy during the velocity pulse. Though the final result looks quite similar to the case with a single saddle, its derivation has some characteristic differences and we present it in detail. Unlike the case with a single saddle, the pulse may start close either to the left turning point or to the right turning point and the sign of the velocity in such pulses is opposite [4,24]. As concerns the angle $\psi$ in the pulse, it is close to $-\pi / 2$ or $\pi / 2$, respectively. So, let us calculate the change of energy from the beginning of the pulse, $t_{k}$, until a given instant $t$ within the pulse

$$
\begin{aligned}
\Delta E & =-\int_{t_{k}}^{t} d \tau \dot{q} h \partial V / \partial q=h \int_{t_{k}}^{t} d \tau \dot{q}\left[-\sin (q) \cos \left(\omega_{f} \tau\right)\right] \\
& \simeq h \cos \left(\omega_{f} t_{k}\right) \int_{t_{k}}^{t} d \tau \dot{q}[-\sin (q)]=\left.h \cos \left(\omega_{f} t_{k}\right) \cos (q)\right|_{t_{k}} ^{t} \\
& \simeq h \cos \left(\omega_{f} t_{k}\right)\{\cos [q(t)]-1\} .
\end{aligned}
$$

Here, the third equality assumes adiabaticity while the last equality takes into account that the turning points are close to the maxima of the potential, i.e., close to a multiple of $2 \pi$ (where the cosine is equal to 1 ).

The quantity $\Delta E$ (69) has the maximal absolute value at $q=\pi$. So, we shall further consider

$$
\begin{aligned}
\Delta E_{\max }=-2 h \cos \left(\omega_{f} t_{k}\right) & \equiv-2 h \cos \left(2 j \psi_{k}-\tilde{\psi}_{k}\right) \\
& =(-1)^{j+1} 2 h \cos \left(\widetilde{\psi}_{k}\right) .
\end{aligned}
$$

The last equality takes into account that, as mentioned above, the relevant $\psi_{k}$ is either $-\pi / 2$ or $\pi / 2$.

For the left wing, the value of $\widetilde{\psi}$ at which the chaotic layer of the separatrix map possesses a minimal energy corresponds to the minimum of the resonance separatrix. It is equal to $\pi$ or 0 if the Fourier coefficient $V_{2 j}$ is positive or negative, i.e., for odd or even $j$, respectively [see Eq. (62)]. Thus $\Delta E_{\max }=-2 h$ for any $j$ and, therefore, it does lower the minimal energy of the boundary. We conclude that

$$
\Delta E_{l}^{(j)}\left(\omega_{f}\right) \simeq \Delta E_{l, \mathrm{NR}}^{(j)}\left(\omega_{f}\right)+2 h, \quad \omega_{f} \leq \omega_{\max }^{(j)},
$$

where $\Delta E_{l, \mathrm{NR}}^{(j)}\left(\omega_{f}\right)$ is given by Eqs. (64) and (65). In particular, the maximum of the peak is

$$
\Delta E_{\max }^{(j)} \simeq(4 e+1) 2 h \approx 23.7 h .
$$

The expression (72) confirms the rough estimate (13) and well agrees with simulations [Fig. 6(c)]. At the same time, it differs from the formula which can be obtained from Eq. (10) of [18] [using also Eqs. (1), (3), (8), and (9) of [18]] in the asymptotic limit $h \rightarrow 0$ : the latter gives for $\Delta E_{\max }^{(j)}$ the asymptotic value $32 h$. Though the result [18] (referred also in [23]) provides for the correct functional dependence on $h$, it is quantitatively incorrect because (i) it is based on the pendulum approximation of the nonlinear resonance while this approximation is not valid in the vicinity of the separatrix, namely, the shape of the true NR separatrix is distinctly asymmetric (cf. Figs. 3 and 4 from [23] and Fig. 4 above) unlike that one in the pendulum approximation (see the discussion of this issue in Sec. III above), and (ii) it does not take into account the variation of energy during the velocity pulse.

The right wing, analogously to the case of the Duffing oscillator, possesses a bending at $\omega_{f}=\omega_{\text {bend }}^{(j)}$ at which $\Delta E_{r, \mathrm{NR}}^{(j)}=\left|\Delta E_{\text {max }}\right| \equiv 2 h$ that corresponds to the switching of the relevant $\widetilde{\psi}$ by $\pi$. We conclude that

$$
\begin{gathered}
\Delta E_{r}^{(j)}\left(\omega_{f}\right)=\Delta E_{r, \mathrm{NR}}^{(j)}\left(\omega_{f}\right), \quad \omega_{\max }^{(j)}<\omega_{f} \leq \omega_{\text {bend }}^{(j)}, \\
\Delta E_{r}^{(j)}\left(\omega_{f}\right)=2 h, \quad \omega_{f} \geq \omega_{\text {bend }}^{(j)}, \\
\omega_{\text {bend }}^{(j)}=\frac{2 \pi j}{\ln (16 / h)-3},
\end{gathered}
$$

where $\Delta E_{r, \mathrm{NR}}^{(j)}\left(\omega_{f}\right)$ is given by Eqs. (66) and (67). Similarly to the previous case, both the peaks and the frequency ranges far beyond the peaks are well approximated by Eq. (41) with $\Delta E_{l}^{(j)}$ and $\Delta E_{r}^{(j)}$ given by Eqs. (71) and (73), respectively [Fig. 6(a)].

\section{ESTIMATE OF THE NEXT-ORDER CORRECTIONS}

We have explicitly calculated only the leading term $\Delta E_{0}^{(j)}$ in the asymptotic expansion of the chaotic layer width. The explicit calculation of the next-order term $\Delta E_{n e x t}^{(j)}$ is possible but it is rather complicated and cumbersome: see the closely related case with two separatrices [24], where most of the next-order contributions are calculated quantitatively [38]. In the present paper, where the perturbation amplitude $h$ in the numerical examples is 4 orders of magnitude smaller than that in [24], there is no particular need to calculate the nextorder term quantitatively. Let us estimate it just qualitatively, with the main purpose to demonstrate that its ratio to the lowest-order term does vanish in the asymptotic limit $h \rightarrow 0$.

One of the contributions to the next-order term stems from the variation of energy during the velocity pulse (in other words, in between instants relevant to the SM). Let us denote it as $\Delta E_{n e x t, p}^{(j)}$. For systems of type I, the variation of energy during the pulse is $\sim h$ while the leading-order term is $\sim h \ln (1 / h) / j$ (see Sec. IV above), so that $\left|\Delta E_{\text {next, }}^{(j)} / \Delta E_{0}^{(j)}\right|$ $\sim j / \ln (1 / h)$. For systems of type II, the variation during the pulse does contribute to the leading-order term $\sim h$ (see Sec. $\mathrm{V}$ above) while the correction to its leading-order expression is $\sim j h / \ln (1 / h)$ (it stems in particular from the deviation from the complete adiabaticity). Thus, for systems of both types,

$$
\left|\frac{\Delta E_{\text {next,p }}^{(j)}}{\Delta E_{0}^{(j)}}\right| \sim \frac{j}{\ln (1 / h)}, \quad j \ll \ln (1 / h) .
$$

Let us turn to the contribution $\Delta E_{n e x t, w}^{(j)}$ stemming from the various corrections within the resonance approximation (16). It may be estimated similarly to the case considered in [24]: it stems from the deviation of the Fourier components $q_{j}$ and $V_{j}$ from their asymptotic values [given by Eqs. (18), (46), and (62) for the corresponding systems], from the difference 
between the exact resonance condition (20) and the approximate one (21), etc. [39]. It can be shown that the absolute value of the ratio between $\Delta E_{n e x t, w}^{(j)}$ and the leading term is logarithmically small in $h$ and, roughly speaking, proportional to $j$ if $j \ll \ln (1 / h)$ (cf. [24]),

$$
\left|\frac{\Delta E_{\text {next,w }}^{(j)}}{\Delta E_{0}^{(j)}}\right| \sim \frac{j}{\ln (1 / h)}, \quad j \ll \ln (1 / h) .
$$

Let us turn to the analysis of the contribution $\Delta E_{n e x t, t}^{(j)}$ stemming from the corrections to the resonance Hamiltonian (16). It is convenient to consider separately the cases of the left and right wings of the peak.

As described in Secs. IV and V above, the left wing corresponds in the leading-order approximation to formation of the boundary of the layer by the separatrix of the resonance Hamiltonian (16). The resonance approximation (16) neglects time-periodic terms while the frequencies of oscillation of these terms greatly exceed the frequency of eigenoscillation of the resonance Hamiltonian (16) around its relevant elliptic point, i.e., the elliptic point inside the area limited by the resonance separatrix. As is well known $[3-6,8,9]$, fast-oscillating terms acting on a system with a separatrix give rise to the onset of an exponentially narrow chaotic layer in place of the separatrix. In the present context, this means that the correction to the maximal action $\tilde{I}$ stemming from fast-oscillating corrections to the resonance Hamiltonian is exponentially small and therefore $\left|\Delta E_{n e x t, t}^{(j)}\right|$ is exponentially small in comparison to $\left|\Delta E_{n e x t, p}^{(j)}\right|$ and $\left|\Delta E_{n e x t, w}^{(j)}\right|$.

The right wing, described in Secs. IV and V above, corresponds in leading-order approximation to the formation of the boundary of the layer by the resonance trajectory tangent to the GSS curve. For the part of the right wing exponentially close in frequency to the frequency of the maximum, the tangent trajectory is close to the resonance separatrix, so that the correction stemming from fast-oscillating terms is exponentially small, similarly to the case of the left wing. As the frequency further deviates from the frequency of the maximum, the tangent trajectory further deviates from the resonance separatrix and the correction $\Delta E_{\text {next }, t}^{(j)}$ differs from the exponentially small correction estimated above. It may be estimated in the following way.

It follows from the second-order approximation of the averaging method [40] that the fast-oscillating terms lead, in the second-order approximation, to the onset of additional terms $h^{2} T_{\tilde{I}}^{-}(\tilde{I}, \widetilde{\psi})$ and $h^{2} T_{\tilde{\psi}}(\tilde{I}, \widetilde{\psi})$ in the dynamic equations for slow variables $\widetilde{I}$ and $\widetilde{\psi}$, respectively, where $T_{\tilde{I}}(\widetilde{I}, \widetilde{\psi})$ and $T_{\tilde{\psi}}(\widetilde{I}, \widetilde{\psi})$ in the relevant range of $\widetilde{I}$ are of the order of powerlaw-like functions of $1 / \ln (1 / h)$. The corresponding correction to the width of the chaotic layer in energy may be expressed as

$$
\Delta E_{n e x t, t}^{(j)}=\int_{t_{\min }}^{t_{\max }} d t h^{2} T_{I}^{\sim} \omega(\widetilde{I}),
$$

where $t_{\min }$ and $t_{\max }$ are instants corresponding to the minimum and maximum deviations of the tangent trajectory from the separatrix of the unperturbed system [cf. Figs. 1(c) and 4(c)]. The interval $t_{\max }-t_{\min }$ may be estimated as follows:

$$
t_{\max }-t_{\min } \sim \frac{\pi}{|\langle\dot{\widetilde{\psi}}\rangle|},
$$

where $\langle\dot{\tilde{\psi}}\rangle$ is the value of $\dot{\widetilde{\psi}}$ averaged over the tangent trajectory. It follows from Eq. (16) that

$$
|\langle\dot{\tilde{\psi}}\rangle| \sim \omega_{f}-\omega\left(E_{s}-\delta\right) \sim \frac{\omega\left(E_{s}-\delta\right)}{\ln (1 / h)} \sim \frac{\omega_{0}}{\ln ^{2}(1 / h)} .
$$

Taking together Eqs. (76)-(78) and allowing for the fact that $T_{I}$ is of the order of a power-law-like function of $1 / \ln (1 / h)$, we conclude that

$$
\Delta E_{n e x t, t}^{(j)} \sim h^{2} P[\ln (1 / h)],
$$

where $P(x)$ is some power-law-like function. The absolute value of $\Delta E_{n e x t, t}^{(j)}$ is still asymptotically smaller than $\left|\Delta E_{n e x t, p}^{(j)}\right|$ and $\left|\Delta E_{\text {next, } w}^{(j)}\right|$, which are of the order of $h$ or $j h / \ln (1 / h)$ for systems of type I or type II respectively.

Thus, we conclude that, both for the left and right wings of the peak, (i) the correction $\Delta E_{n e x t}^{(j)}$ is determined by the corrections $\Delta E_{\text {next,p }}^{(j)}$ and $\Delta E_{\text {next,w }}^{(j)}$ and (ii) in the asymptotic limit $h \rightarrow 0$, the overall next-order correction is negligible in comparison to the leading term for any given $j$,

$$
\begin{aligned}
\left|\frac{\Delta E_{\text {next }}^{(j)}}{\Delta E_{0}^{(j)}}\right| & \equiv\left|\frac{\Delta E_{\text {next }, p}^{(j)}+\Delta E_{\text {next }, w}^{(j)}+\Delta E_{\text {next, }}^{(j)}}{\Delta E_{0}^{(j)}}\right| \\
& \approx\left|\frac{\Delta E_{\text {next }, p}^{(j)}+\Delta E_{\text {next }, w}^{(j)}}{\Delta E_{0}^{(j)}}\right| \sim \frac{j}{\ln (1 / h)} \rightarrow 0 .
\end{aligned}
$$

This estimate well agrees with results in Figs. 3-6.

\section{DISCUSSION}

In this section, we briefly discuss the following issues: (1) scaled asymptotic shape of the peaks, (2) peaks in the range of moderate frequencies, (3) jumps in the amplitude dependence of the layer width, and (4) an application to the global chaos onset.

(1) Let us analyze the scaled asymptotic shape of the peaks. Consider first systems of type I. The peaks are described in the leading-order approximation exclusively within the separatrix map dynamics (approximated, in turn, by the NR dynamics). Let us assume that $1-\omega_{f} / \omega_{\max }^{(j)} \ll 1$ (the relevance of this condition will be clear further down), keep only the leading-order term in Eq. (36) for $\Delta E_{l}^{(j)}\left(\omega_{f}\right)$, as well as express $h$ from Eq. (34). Besides, let us express $h /[(2 j-1) \pi]$ from Eq. (26), substitute it in Eq. (32) for $y$, and keep only the leading-order terms both in the exponent and in the prefactor. Then, $\Delta E_{l}^{(j)}\left(\omega_{f}\right)=\Delta E_{\max }^{(j)} S_{l}(\alpha)$, where $\alpha$ and $S_{l}(\alpha)$ are defined in Eqs. (81) and (82), respectively. Similarly, we can express $\Delta E_{r}^{(j)}\left(\omega_{f}\right)$ using Eqs. (40), (34), (39), and (26). Thus, most of the peak with a given $j$ can be written in the universal scaled form

$$
\Delta E^{(j)}\left(\omega_{f}\right)=\Delta E_{\max }^{(j)} S\left(\frac{\pi(2 j-1)}{\left(\omega_{\max }^{(j)}\right)^{2}}\left(\omega_{f}-\omega_{\max }^{(j)}\right)\right),
$$




$$
\left|\frac{\omega_{f}}{\omega_{\max }^{(j)}}-1\right| \ll 1,
$$

where the universal function $S(\alpha)$ is strongly asymmetric

$$
\begin{gathered}
S(\alpha)=\left\{\begin{array}{l}
S_{l}(\alpha) \text { for } \quad \alpha \leq 0 \\
S_{r}(\alpha) \text { for } \quad \alpha>0,
\end{array}\right. \\
S_{l}(\alpha)=\frac{1}{e[\ln (1+y)-y /(1+y)]}, \\
(1+y) \ln (1+y)-y=\exp (-\alpha), \\
S_{r}(\alpha)=\frac{1}{e[1+\ln (1 / z)]}, \\
z[1+\ln (1 / z)]=\exp (-\alpha), \\
|\alpha| \ll \ln \left(\frac{8(2 j-1)}{h}\right) .
\end{gathered}
$$

Allowing for the expression for $\alpha$ relevant to Eq. (81), one can see that the strong inequality in the last line of Eq. (82) corresponds to the strong inequality in Eq. (81). Note also that, as follows from Eqs. (81), (82), and (35), if the strong inequality in Eq. (81) is not satisfied, then $\Delta E^{(j)}$ falls down to values $\lesssim h$ and therefore the leading-order approximation developed in the present paper becomes invalid.

It is not difficult to show that

$$
\begin{gathered}
S_{l}(\alpha=0)=1, \quad S_{r}(\alpha \rightarrow+0)=e^{-1}, \\
\frac{d S_{l}(\alpha=0)}{d \alpha}=1-e^{-1}, \quad \frac{d S_{r}(\alpha \rightarrow+0)}{d \alpha} \rightarrow-\infty, \\
S(\alpha) \propto \frac{1}{|\alpha|} \quad \text { for } \quad|\alpha| \gg 1 .
\end{gathered}
$$

Thus, the function $S(\alpha)$ is discontinuous at the maximum. To the left of the maximum, the function relatively slowly approaches the far part of the wing (which falls down as $1 /|\alpha|$ ) while, to the right of the maximum, the function first drops jump-wise by a factor $e$ and then sharply approaches the far part of the wing (which falls down as $1 / \alpha$ ).

As follows from Eqs. (81)-(83) and (27), the peaks are logarithmically narrow, i.e., the ratio of the half width of the peak, $\Delta \omega^{(j)}$, to $\omega_{\max }^{(j)}$ is logarithmically small

$$
\frac{\Delta \omega^{(j)}}{\omega_{\max }^{(j)}} \sim \frac{1}{\ln [8(2 j-1) / h]} .
$$

We emphasize that the shape (82) is not restricted to the example (14): it is valid for any system of type I.

For systems of type II, the contributions from the NR and from the variation of energy during the pulse of velocity, as concerns the $h$ dependence, are formally of the same order but, numerically, the latter contribution is typically much smaller than the former one. Thus, typically, the function (82) well approximates the properly scaled shape of the major part of the peak for systems of type II too.
(2) The quantitative theory presented in the paper relates only to the peaks of small order $n$, i.e., in the range of logarithmically small frequencies. At the same time, the magnitude of the peaks is still significant up to the frequencies of the order of 1 . This occurs because, for the motion close to the separatrix, the order of magnitude of the Fourier coefficients remains significant up to logarithmically large numbers $n$. The shape of the peaks remains the same but their magnitude decreases typically (but, in some cases, it may even increase in some range of frequencies). The quantitative description of this decrease as well as the analysis of more sophisticated cases requires a generalization of our theory, which will be presented elsewhere.

(3) Apart from the frequency dependence of the layer width, our theory is also relevant for the amplitude dependence: it describes the jumps [20] in the dependence of the width on $h$ and the transition between the jumps and the linear dependence. The values of $h$ at which the jumps occur, $h_{\text {jump }}^{(j)}$, are determined by the same condition which determines $\omega_{\max }^{(j)}$ in the frequency dependence of the width. The formulas relevant to the left wings of the peaks in the frequency dependence describe the ranges $h>h_{j u m p}^{(j)}$ while the formulas relevant to the right wings describe the ranges $h$ $<h_{\text {jump }}^{(j)}$

(4) Finally, we note that, apart from systems with a separatrix, our work may be relevant to nonlinear resonances in any system. If the system is perturbed by a weak timeperiodic perturbation, then nonlinear resonances arise and their dynamics is described by the model of the auxiliary time-periodically perturbed pendulum [2-8]. If the original perturbation has a single harmonic, then the effective perturbation of the auxiliary pendulum is necessarily a highfrequency one and chaotic layers associated with the resonances are exponentially narrow [2-8] while our results are irrelevant. But, if either the amplitude or the angle of the original perturbation is slowly modulated or if there is an additional harmonic of a slightly shifted frequency, then the effective perturbation of the auxiliary pendulum is a lowfrequency one [24] and the layers become much wider [41] while our theoretical approach becomes relevant. It may allow to find optimal parameters of the perturbation for the facilitation of the onset of global chaos associated with the overlap in energy between different-order nonlinear resonances [2]: the overlap may be expected to occur at a much smaller amplitude of perturbation in comparison to that one required for the overlap in case of a single-harmonic perturbation.

\section{CONCLUSIONS}

We have further developed an approach [24] to the treatment of the separatrix chaos in the range of logarithmically small frequencies of a weak time-periodic perturbation, where the chaos typically takes the largest possible area in phase space. The approach is based on the matching between the discrete chaotic dynamics of the separatrix map and the continuous regularlike dynamics of the resonance Hamiltonian. Using this approach and taking also into account the dynamics in between instants corresponding to the separatrix 
map, i.e., during velocity pulses, we have presented an accurate asymptotic description of high sharp peaks of the width of the separatrix chaotic layer in energy as function of the frequency of a weak time-periodic perturbation, including in particular the absolute maximum of the function. Our work provides the accurate base to explain former numerical and heuristic results and intuitive assumptions [18,20,22-25] and shows that the previous heuristic theory [18] for the pendulum in which the suspension point oscillates with an amplitude $h$ gives for the heights of the peaks an inaccurate result when it is applied to the asymptotic limit $h \rightarrow 0$. Our theory also discovers important features and opens up horizons for future studies and applications.

The observed peaks arise due to the involvement of the nonlinear resonance dynamics into the separatrix chaotic motion and the mechanism of this involvement is explained by us. In the context of the heights of the peaks, all systems are classified into two types: the heights of the peaks are proportional to the perturbation amplitude $h$ times either a logarithmically large factor $\propto \ln (1 / h)$ (for systems of type I) or a numerical factor (for systems of type II). Type I includes systems for which the separatrix of their unperturbed Hamiltonian has more than one saddle while the perturbation is not identical on adjacent saddles. All other systems belong to type II. The detailed theory is developed for three archetypal examples: the ac-driven pendulum (type I), the ac-driven Duffing oscillator (type II), and the pendulum with the oscil- lating suspension point (type II). The theory is verified by computer simulations.

The shape of the peaks is strongly asymmetric. In the asymptotic limit of small amplitudes, the shape of the peaks for type I is universal. For type II, the shape is quite similar, differing only by a typically small contribution stemming from the variation of energy during the velocity pulse.

Our theory describes the jumps of the width as a function of the perturbation amplitude $h$ as well as the transition between the jumps and the linear dependence. Finally, our work suggests a method for the facilitation of global chaos onset due to the enhanced overlap of nonlinear resonances. The theoretical approach developed by us may be used to derive the optimal choice of parameters of the perturbation leading to the facilitation.

\section{ACKNOWLEDGMENTS}

This work was partly supported by the grant within the Convention between the Institute of Semiconductor Physics and University of Pisa for 2008-2009 and by the Royal Society Grant No. 2007/R2-IJP. We acknowledge discussions with Vassili Gelfreich, Igor Khovanov, Ivan Shevchenko, and Oleg Yevtushenko. We especially appreciate numerous stimulating discussions with the late George Zaslavsky and his role for the subject of Hamiltonian chaos on the whole.
[1] G. M. Zaslavsky and N. N. Filonenko, Sov. Phys. JETP 27, 851 (1968)

[2] B. V. Chirikov, Phys. Rep. 52, 263 (1979).

[3] A. J. Lichtenberg and M. A. Lieberman, Regular and Stochastic Motion (Springer, New York, 1992).

[4] G. M. Zaslavsky, R. D. Sagdeev, D. A. Usikov, and A. A. Chernikov, Weak Chaos and Quasi-Regular Patterns (Cambridge University Press, London, 1991).

[5] G. M. Zaslavsky, Physics of Chaos in Hamiltonian systems (Imperial Colledge Press, London, 2007).

[6] G. M. Zaslavsky, Hamiltonian Chaos and Fractional Dynamics (Oxford University Press, New York, 2008).

[7] S. S. Abdullaev, Construction of Mappings for Hamiltonian Systems and Their Applications (Springer, Heidelberg, 2006).

[8] V. G. Gelfreich and V. F. Lazutkin, Russ. Math. Surveys 56, 499 (2001).

[9] G. N. Piftankin and D. V. Treschev, Russ. Math. Surveys 62, 219 (2007).

[10] L. E. Reichl, The Transition to Chaos in Conservative Classical Systems: Quantum Manifestations (Springer, New York, 1992).

[11] Chaos and Stability in Planetary Systems, edited by R. Dvorak, F. Freistetter, and J. Kurths, Lecture Notes in Physics (Springer, Berlin, 2005), Vol. 683.

[12] A. I. Neishtadt, Sov. J. Plasma Phys. 12, 568 (1986).

[13] Y. Elskens and D. F. Escande, Nonlinearity 4, 615 (1991).

[14] A. I. Neishtadt, V. V. Sidorenko, and D. V. Treschev, Chaos 7, 2 (1997).
[15] S. M. Soskin, O. M. Yevtushenko, and R. Mannella, Phys. Rev. Lett. 95, 224101 (2005).

[16] S. M. Soskin, O. M. Yevtushenko, and R. Mannella, Commun. Nonlinear Sci. Numer. Simul. 15, 19 (2009).

[17] V. Gelfreich (private communication).

[18] I. I. Shevchenko, Phys. Scr. 57, 185 (1998).

[19] A. C. J. Luo, K. Gu, and R. P. S. Han, Nonlinear Dyn. 19, 37 (1999).

[20] S. M. Soskin, R. Mannella, M. Arrayás, and A. N. Silchenko, Phys. Rev. E 63, 051111 (2001).

[21] A. C. J. Luo, Appl. Mech. Rev. 57, 161 (2004).

[22] V. V. Vecheslavov, Tech. Phys. 49, 521 (2004).

[23] I. I. Shevchenko, Phys. Lett. A 372, 808 (2008).

[24] S. M. Soskin, R. Mannella, and O. M. Yevtushenko, Phys. Rev. E 77, 036221 (2008).

[25] S. M. Soskin, R. Mannella, and O. M. Yevtushenko, in Chaos, Complexity and Transport: Theory and Applications, Proceedings of the CCT-07, edited by C. Chandre, X. Leoncini, and G. Zaslavsky (World Scientific, Singapore, 2008), pp. 119-128.

[26] V. Rom-Kedar, Physica D 43, 229 (1990).

[27] Formally, $\operatorname{sgn}\left(E-E_{s}\right)$ is not defined for $E=E_{s}$ but, if we shifted $E$ from $E_{s}$ by an infinitesimal value, $\operatorname{sgn}\left(E-E_{s}\right)$ would acquire a value equal to either +1 or -1 , depending on the sign of the shift. Given that $\sigma_{i}$ is proportional to $\operatorname{sgn}\left(E_{s}-E_{i}\right)$ while the latter is randomlike (as it has been shown above), $\sigma_{i+1}$ is not correlated with $\sigma_{i}$ if $E_{i+1}=E_{s} \pm 0$.

[28] For the SM relating to the ac-driven spatially periodic systems, the time during which the SM undergoes a regularlike evolu- 
tion above the separatrix diverges in the adiabatic limit $\omega_{f}$ $\rightarrow 0$ [16] and the width of the part of the SM layer above the separatrix diverges too. However, we do not consider this case here since it is irrelevant to the main subject of the present paper, i.e., to the involvement of the resonance dynamics into the separatrix chaotic motion.

[29] The GSS curve corresponds to the step of the SM which follows the state with $E=E_{s}$ as described above.

[30] The interval which is relevant is the one for which the derivative $d E / d t$ in the nonlinear resonance equations [see Eq. (16) below] is positive or negative for the case of the upper or lower GSS curve, respectively.

[31] Namely $E$ determined by Eq. (7) for any $\tilde{\psi}$ except from the close vicinity of multiples of $\pi$. As shown in [24], Eq. (7) is irrelevant to the boundary of the chaotic layer in the range of $\widetilde{\psi}$ close to multiples of $\pi$ while the boundary in this range of $\widetilde{\psi}$ still lies in the resonance range of energies, where $\omega(E)$ $\approx \omega_{r}^{( \pm)}$.

[32] As an example of the heuristic estimate, we use the formula from [4]: $\Delta E^{(-)} / h=2 \pi \omega_{f} / \cosh \left(\pi \omega_{f} / 2\right)$.

[33] The adiabatic estimate for $\Delta E^{(-)}\left(\omega_{f}\right)$ is equal approximately to $2 \pi$. The estimate following from the results of the mathematical work [9] for $\omega_{f} \sim 1$ is of the same order, so that it is conditionally represented in the inset in Fig. 2 by the same line as for the adiabatic estimate (dashed line).

[34] S. M. Soskin, R. Mannella, and P. V. E. McClintock, Phys. Rep. 373, 247 (2003).

[35] M. I. Dykman, S. M. Soskin, and M. A. Krivoglaz, Physica A 133, 53 (1985).
[36] The only essential difference consists in that $q_{n}$ at the saddle is described by Eq. (46) rather than by Eq. (18).

[37] The disagreement between theory and simulations for the height of the second peak is about twice larger than that for the first peak, so that the height of the second peak is about $30 \%$ smaller than that by the asymptotic theory. This occurs mainly because the (negative) deviation of the Fourier coefficient $q_{2}$ from the asymptotic formula (42) is about twice larger than that for $q_{1}$. Besides, the velocity pulse contribution $\Delta E$ also significantly decreases while the separatrix split increases as $\omega_{f}$ becomes $\sim 1$. The generalization of the theory for $\omega_{f} \sim 1$ will be presented elsewhere.

[38] The paper [24] quantitatively calculates all contributions to the next-order correction except the contribution stemming from the second-order approximation of the averaging method [40], i.e., from the next-order correction to the resonance Hamiltonian.

[39] Note that the correction stemming from the deviation of the GSS curve from the separatrix does not contribute to the nextorder term $\Delta E_{\text {next,w }}^{(j)}$ since it is smaller than the latter by the factor $\sim \sqrt{\ln (1 / h)}$ (cf. [24]) which diverges in the asymptotic limit $h \rightarrow 0$.

[40] N. N. Bogolyubov and Yu. A. Mitropolsky, Asymptotic Methods in the Theory of Nonlinear Oscillators (Gordon and Breach, New York, 1961).

[41] This should not be confused with the widening occurring with the separatrix chaotic layer in the original pendulum if an originally single-harmonic perturbation of a high frequency is completed by one more harmonic of a slightly shifted frequency (see [22] and references therein). 